\title{
The notion of cusp forms for a class of reductive symmetric spaces of split rank 1
}

\author{
Erik P. van den Ban, Job J. Kuit, and Henrik Schlichtkrull
}

\begin{abstract}
We study a notion of cusp forms for the symmetric spaces $G / H$ with $G=$ $\mathrm{SL}(n, \mathbb{R})$ and $H=\mathrm{S}(\mathrm{GL}(n-1, \mathbb{R}) \times \mathrm{GL}(1, \mathbb{R}))$. We classify all minimal parabolic subgroups of $G$ for which the associated cuspidal integrals are convergent and discuss the possible definitions of cusp forms. Finally, we show that the closure of the direct sum of the discrete series representations of $G / H$ coincides with the space of cusp forms.
\end{abstract}

\section{Introduction}

In this article we investigate the convergence of certain integrals that can be used to give a notion of cusp forms on the symmetric space $\operatorname{SL}(n, \mathbb{R}) / \mathrm{S}(\mathrm{GL}(n-1, \mathbb{R}) \times$ $\operatorname{GL}(1, \mathbb{R}))$, which we here denote by $X_{n}$. Furthermore, we determine the relation between the discrete series representations and the space of cusp forms for these spaces.

Harish-Chandra defined a notion of cusp forms for reductive Lie groups and proved that the space of cusp forms coincides with the closed span in the $L^{2}$ Schwartz space of the discrete series representations. This fact plays an important role in his work on the Plancherel decomposition. In [1] a notion of cusp forms for real hyperbolic spaces was introduced, following a more general suggestion by M. Flensted-Jensen. Subsequently, in [4], the first and second author gave a definition of cusp forms for split rank 1 reductive symmetric spaces. However, the notion of [4] deviates from the general suggestion of Flensted-Jensen at an important point. The main purpose of the present article is to explore the necessity of this deviation.

In order to give a precise description of the purpose of the present article we first recall some background. Let $G$ be semisimple, and let $G / H$ be a symmetric space of split rank 1; here $H$ is an open subgroup of the group of fixed points of an involution $\sigma$ of $G$. Every minimal parabolic subgroup $P$ of $G$ contains a $\sigma$-stable maximal split connected abelian subgroup $A_{P}$ of $G$. The set of minimal parabolic subgroups decomposes into two disjoint sets: the set $\mathcal{P}$ of $P$ such that

Kyoto Journal of Mathematics, Vol. 59, No. 2 (2019), 471-513

First published online May 8, 2019.

DOI 10.1215/21562261-2019-0015, C 2019 by Kyoto University

Received January 26, 2016. Accepted April 03, 2017.

2010 Mathematics Subject Classification: Primary 22E30; Secondary 22E46, 43A80. 
$A_{P} /\left(A_{P} \cap H\right)$ is 1 -dimensional, and the set $\mathcal{Q}$ of $P$ such that $A_{P} /\left(A_{P} \cap H\right)$ is 0-dimensional, that is, $A_{P} \subseteq H$.

The main goal is now to identify a suitable class of minimal parabolic subgroups $P$ with the property that for every $\phi$ in the Harish-Chandra Schwartz space $\mathcal{C}(G / H)$ the integral

$$
\int_{N_{P} /\left(N_{P} \cap H\right)} \phi(n) d n
$$

is absolutely convergent. Here $N_{P}$ denotes the unipotent radical of $P$, and $d n$ is an $N_{P}$-invariant Radon measure on $N_{P} /\left(N_{P} \cap H\right)$. A cusp form is then defined to be a function $\phi \in \mathcal{C}(G / H)$ such that

$$
\int_{N_{P} /\left(N_{P} \cap H\right)} \phi(g n) d n=0
$$

for every such parabolic subgroup $P$ and every $g \in G$.

Flensted-Jensen has suggested using the set of parabolic subgroups that can be characterized as

$$
\mathcal{P}_{*}:=\left\{P \in \mathcal{P}: \operatorname{dim}\left(N_{P} \cap H\right)=\max _{Q \in \mathcal{P}} \operatorname{dim}\left(N_{Q} \cap H\right)\right\} .
$$

These parabolic subgroups are said to be $\mathfrak{h}$-extreme (see [4, Definition 1.1] for an equivalent characterization).

In [1] it was confirmed for real hyperbolic spaces that the integral is absolutely convergent for $P \in \mathcal{P}_{*}$ and for every $\phi \in \mathcal{C}(G / H)$.

In [4] a notion called $\mathfrak{h}$-compatibility was introduced for parabolic subgroups $P \in \mathcal{P}$ by imposing a condition on the roots that are positive for $P$. For the spaces $X_{n}$ this condition is recalled in Definition 2.5. It was proved in [4] that for $\mathfrak{h}$ compatible parabolic subgroups $P \in \mathcal{P}$ the integral (I.1) is absolutely convergent. Let

$$
\mathcal{P}_{\mathfrak{h}}:=\{P \in \mathcal{P}: P \text { is } \mathfrak{h} \text {-compatible }\} .
$$

For real hyperbolic spaces this set equals $\mathcal{P}_{*}$, but in general this need not be the case. The difference occurs for example for the symmetric spaces $X_{n}$. If $n \geq 4$, then $\mathcal{P}_{*}$ is not contained in $\mathcal{P}_{\mathfrak{h}}$, and if $n \geq 3$ is odd, then $\mathcal{P}_{\mathfrak{h}}$ is not contained in $\mathcal{P}_{*}$. Therefore, the family $X_{n}$ is a good test case for determining whether or not the need for $\mathfrak{h}$-compatible parabolic subgroups in [4] is an artifact of the proof, and whether or not the dimension of $N_{P} \cap H$ is relevant for the convergence of the integrals.

In Section 1 we describe some generalities concerning parabolic subgroups. The results in this section hold for any reductive symmetric space. In Section 2 we describe the polar decomposition of $X_{n}$ and the Harish-Chandra Schwartz space $\mathcal{C}\left(X_{n}\right)$ of $X_{n}$. Our main results are formulated in Section 3 and proved in the remaining sections.

The first main result (Theorem 3.1) is a classification of all minimal parabolic subgroups $P$ such that the integral (I.1) is absolutely convergent for all $\phi \in \mathcal{C}\left(X_{n}\right)$. We extend the notion of $\mathfrak{h}$-compatibility to all minimal parabolic 
subgroups $P$ (not just those in $\mathcal{P}$, but also the ones in $\mathcal{Q}$ ). We then show that (I.1) is absolutely convergent for all $\phi \in \mathcal{C}\left(X_{n}\right)$ if and only if $P$ is $\mathfrak{h}$-compatible.

The second main result (Theorem 3.2) describes the behavior at infinity of the function

$$
\mathcal{H}_{P} \phi: A_{P} \rightarrow \mathbb{C}, \quad a \mapsto a^{\rho_{P}} \int_{N_{P} /\left(N_{P} \cap H\right)} \phi(a n) d n,
$$

for $P \in \mathcal{P}_{\mathfrak{h}} \cap \mathcal{P}_{*}$ and $\phi \in \mathcal{C}\left(X_{n}\right)$. In particular, it is shown that if $n$ is even, then $\mathcal{H}_{P} \phi$ is rapidly decreasing; if $n$ is odd, then $\mathcal{H}_{P} \phi$ is rapidly decreasing in one direction, while it converges to a possibly nonzero limit in the other direction. Moreover, for every $\mathfrak{h}$-compatible $P \in \mathcal{Q}$ there exist a $P^{\prime} \in \mathcal{P}_{\mathfrak{h}} \cap \mathcal{P}_{*}$ and a $g \in G$ such that the integral (I.1) equals a limit of $\mathcal{H}_{P^{\prime}}(\phi(g \cdot))$.

As a consequence of Theorem 3.2, we will show in Proposition 3.3 that if (I.2) holds for all $P \in \mathcal{P}_{\mathfrak{h}} \cap \mathcal{P}_{*}$, then (I.2) holds for all $\mathfrak{h}$-compatible parabolic subgroups. This and Theorem 3.1 justify the use of $\mathcal{P}_{\mathfrak{h}} \cap \mathcal{P}_{*}$ in the definition of cusp forms for reductive symmetric spaces of split rank 1 .

In [1] it was shown that there exist discrete series representations for certain real hyperbolic spaces for which the generating functions are not cusp forms. These discrete series representations are called noncuspidal. Using the results of [4] and the estimates from Theorem 3.2 for the behavior at infinity of (I.3), we show that for $X_{n}$ there are no noncuspidal discrete series representations. Our final main result (Theorem 3.6) is thus that the space of cusp forms on $X_{n}$ coincides with the closed span of the discrete series representations for $X_{n}$. For other papers concerning the particular symmetric space $X_{n}$, see, for example, [12], [11], [6], [7], and [16].

\section{Parabolic subgroups and split components}

In this preliminary section we collect some properties which are valid for general reductive symmetric spaces $G / H$. Here $G$ is a reductive Lie group of the HarishChandra class, and $H$ is an open subgroup of the group of fixed points for an involution $\sigma$ of $G$. We are concerned with properties of $\sigma$-stable connected split abelian subgroups and parabolic subgroups of $G$. The main result is that every parabolic subgroup $P$ of $G$ contains a $\sigma$-stable maximal split abelian subgroup $A$ of $G$, which is unique up to conjugation by an element of $N_{P} \cap H$.

\subsection{Split abelian subalgebras}

We write $\mathfrak{g}=\mathfrak{h} \oplus \mathfrak{q}$ for the eigenspace decomposition for the infinitesimal involution $\sigma$. Here $\mathfrak{h}$ is the Lie algebra of $H$, and $\mathfrak{q}$ is the -1 eigenspace. Recall that an abelian subspace $\mathfrak{a}$ of $\mathfrak{g}$ is called split if $\mathfrak{g}$ decomposes as a sum of joint $\mathfrak{a}$-weight spaces.

LEMMA 1.1

Let $\mathfrak{a}$ be a $\sigma$-stable maximal split abelian subalgebra of $\mathfrak{g}$. Then there exists a $\sigma$-stable Cartan decomposition $\mathfrak{g}=\mathfrak{k} \oplus \mathfrak{p}$ with $\mathfrak{a} \subseteq \mathfrak{p}$. 
Proof

From the construction in [10, Theorems 6.10 and 6.11] it follows that there exists a Cartan involution $\theta_{0}$ such that $\theta_{0}(X)=-X$ for $X \in \mathfrak{a}$. Since $\mathfrak{a}$ is $\sigma$-stable, the construction in the proof of [8, Theorem III.7.1], applied to $\theta_{0}$ and $\sigma$, gives a Cartan involution $\theta$ which in addition commutes with $\sigma$.

\section{DEFINITION 1.2}

We define $\mathcal{A}_{\mathfrak{q}}$ to be the set of all maximal split abelian subspaces $\mathfrak{b}$ of $\mathfrak{q}$, and we define $\mathcal{A}$ to be the set of all maximal split abelian subalgebras $\mathfrak{a}$ of $\mathfrak{g}$ for which $\mathfrak{a} \cap \mathfrak{q} \in \mathcal{A}_{\mathfrak{q}}$. The split rank of $G / H$ is the dimension of any $\mathfrak{b} \in \mathcal{A}_{\mathfrak{q}}$. (It will follow from Corollary 1.5 below that this is well defined.)

Note that $H$ acts on $\mathcal{A}_{\mathfrak{q}}$ and $\mathcal{A}$ by conjugation.

\section{LEMMA 1.3}

If $\mathfrak{a} \in \mathcal{A}$, then $\mathfrak{a}$ is $\sigma$-stable.

Proof

Let $Y \in \mathfrak{a}$. Then $Y$ and $\sigma Y$ both commute with $\mathfrak{a} \cap \mathfrak{q}$, and hence, $Y-\sigma Y \in \mathfrak{a} \cap \mathfrak{q}$ by maximality. Hence, $\sigma Y \in \mathfrak{a}$.

\section{PROPOSITION 1.4}

A subspace $\mathfrak{a} \subseteq \mathfrak{g}$ belongs to $\mathcal{A}$ if and only if there exists a $\sigma$-stable Cartan decomposition $\mathfrak{g}=\mathfrak{k} \oplus \mathfrak{p}$ such that $\mathfrak{a}$ is maximal abelian in $\mathfrak{p}$ and $\mathfrak{a} \cap \mathfrak{q}$ is maximal abelian in $\mathfrak{p} \cap \mathfrak{q}$. The action of $H$ on $\mathcal{A}$ by conjugation is transitive.

\section{Proof}

Let $\mathcal{A}^{\prime}$ denote the set of subspaces $\mathfrak{a} \subseteq \mathfrak{g}$ for which there exists a $\sigma$-stable Cartan decomposition $\mathfrak{g}=\mathfrak{k} \oplus \mathfrak{p}$ such that $\mathfrak{a}$ is maximal abelian in $\mathfrak{p}$ and $\mathfrak{a} \cap \mathfrak{q}$ is maximal abelian in $\mathfrak{p} \cap \mathfrak{q}$. It follows from [14, Lemmas 4 and 7$]$ that $H$ acts transitively on $\mathcal{A}^{\prime}$.

It follows from Lemmas 1.1 and 1.3 that $\mathcal{A} \subseteq \mathcal{A}^{\prime}$. Hence, $\mathcal{A}=\mathcal{A}^{\prime}$.

\section{COROLLARY 1.5}

A subspace $\mathfrak{b} \subseteq \mathfrak{q}$ belongs to $\mathcal{A}_{\mathfrak{q}}$ if and only if there exists a $\sigma$-stable Cartan decomposition $\mathfrak{g}=\mathfrak{k} \oplus \mathfrak{p}$ such that $\mathfrak{b}$ is a maximal abelian subspace of $\mathfrak{p} \cap \mathfrak{q}$. The action of $H$ on $\mathcal{A}_{\mathfrak{q}}$ by conjugation is transitive.

Proof

Let $\mathfrak{b} \in \mathcal{A}_{\mathfrak{q}}$, and let $\mathfrak{a} \in \mathcal{A}$ with $\mathfrak{b}=\mathfrak{a} \cap \mathfrak{q}$. The asserted Cartan decomposition exists according to Proposition 1.4.

Conversely, let $\mathfrak{b} \subseteq \mathfrak{q}$, and assume that $\mathfrak{b}$ is maximal abelian in $\mathfrak{p} \cap \mathfrak{q}$ for some $\sigma$-stable Cartan decomposition $\mathfrak{g}=\mathfrak{k} \oplus \mathfrak{p}$. Let $\mathfrak{a} \subseteq \mathfrak{p}$ be a maximal abelian subspace with $\mathfrak{a} \supset \mathfrak{b}$. Then $\mathfrak{a} \cap \mathfrak{q}=\mathfrak{b}$ by maximality. It follows from Proposition 1.4 
that $\mathfrak{a} \in \mathcal{A}$ and hence $\mathfrak{b} \in \mathcal{A}_{\mathfrak{q}}$. The transitivity of the action follows from the corresponding statement in Proposition 1.4.

\subsection{Parabolic subgroups}

We recall that if $P$ and $Q$ are parabolic subgroups, then $Q$ is called opposite to $P$ if $P \cap Q$ is a common Levi subgroup of $P$ and $Q$. If $P$ is a parabolic subgroup, we write $N_{P}$ for its unipotent radical. Recall also that a split component of $P$ is a maximal connected split subgroup of the center of a Levi subgroup of $P$.

Note that a parabolic subgroup $P$ is minimal if and only if the Lie algebras of its split components are maximal split in $\mathfrak{g}$. Note also that if $\mathfrak{a}$ is a maximal split abelian subalgebra of $\mathfrak{g}$ and $A=\exp (\mathfrak{a})$, then the normalizer $N_{G}(A)$ of $A$ in $G$ acts by conjugation on the set of minimal parabolic subgroups containing $A$. This action is transitive.

LEMMA 1.6

Let $P$ be a minimal parabolic subgroup. If $A$ and $B$ are two split components of $P$, then there exists a unique $n \in N_{P}$ such that $B=n A n^{-1}$.

Proof

There exists $g \in G$ such that $B=g A g^{-1}$. Then $P$ and $g^{-1} P g$ both contain $A$, and hence, $g^{-1} P g=w P w^{-1}$ for some $w \in N_{G}(A)$. The product $g w$ normalizes $P$ and, thus, belongs to $P$. The existence of $n$ now follows by decomposing this element according to the Levi decomposition $P=Z_{G}(A) N_{P}$.

Now assume that $n_{1}, n_{2} \in N_{P}$ satisfy $B=n_{1,2} A n_{1,2}^{-1}$. Then $n_{1}^{-1} n_{2}$ centralizes $A$, and thus, $n_{1}^{-1} n_{2} \in Z_{G}(A) \cap N_{P}=\{e\}$. This proves uniqueness.

\section{LEMMA 1.7}

Let $P$ and $Q$ be minimal parabolic subgroups. Assume that $P$ and $Q$ have common split components $A$ and $B$. Then there exists a unique $n \in N_{P} \cap N_{Q}$ such that $B=n A n^{-1}$.

\section{Proof}

By Lemma 1.6 there exists a unique $n \in N_{P}$ such that $B=n A n^{-1}$. It suffices to show that $n \in N_{Q}$. Let $a \in A$ be dominant with respect to $P$. Then for every $k \in \mathbb{N}$ we have $a^{-k} n a^{k} n^{-1} \in a^{-k} B \subseteq Q$. Since $Q$ is a closed subgroup and $a^{-k} n a^{k} n^{-1}$ converges to $n^{-1}$ for $k \rightarrow \infty$, it follows that $n \in Q$. Furthermore, since $n \in N_{P}$, the element $\log (n)$ is a sum of $\mathfrak{a}$-weight vectors with nonzero weights. This implies that $n \in N_{Q}$.

\section{THEOREM 1.8}

Let $P$ be a minimal parabolic subgroup.

(i) There exists a $\sigma$-stable split component $A$ of $P$.

(ii) If $A$ and $B$ are two $\sigma$-stable split components of $P$, then there exists a unique $n \in N_{P} \cap H$ such that $B=n A n^{-1}$. 
Proof

See [17, Lemma 12] for a proof that uses [15] and only applies to algebraic groups. An alternative proof is given in [9, Lemma 2.4], also under the assumption of algebraic groups. The statement (i) is proved without this assumption in [14, Lemma 2], and hence we only need to prove (ii).

If $A$ and $B$ are two $\sigma$-stable split components of $P$, then by Lemma 1.7 there exists a unique $n \in N_{P} \cap \sigma\left(N_{P}\right)$ such that $B=n A n^{-1}$. Observe that

$$
B=\sigma(B)=\sigma(n) \sigma(A) \sigma(n)^{-1}=\sigma(n) A \sigma(n)^{-1} .
$$

From the uniqueness of $n$ we conclude that $\sigma(n)=n$, or equivalently, $n \in N_{P} \cap H$.

The preceding theorem allows for the following definition.

\section{DEFINITION 1.9}

Let $P$ be a minimal parabolic subgroup, and let $A$ be a $\sigma$-stable split component of $P$. We define the $\sigma$-parabolic rank of $P$ to be the dimension of $A /(A \cap H)$.

We write $\mathcal{P}$ for the set of minimal parabolic subgroups of maximal $\sigma$-parabolic rank. (Note that $P \in \mathcal{P}$ if and only if $P$ admits a split component $A=\exp \mathfrak{a}$ with $\mathfrak{a} \in \mathcal{A}$.) Furthermore, we write $\mathcal{Q}$ for the set of all minimal parabolic subgroups of minimal $\sigma$-parabolic rank. If $\mathfrak{a} \in \mathcal{A}$, then we write $\mathcal{P}(\mathfrak{a})$ for the set of minimal parabolic subgroups containing exp $\mathfrak{a}$. Note that $\mathcal{P}(\mathfrak{a}) \subseteq \mathcal{P}$. If $\mathfrak{b}$ is a $\sigma$-stable maximal split abelian subalgebra of $\mathfrak{g}$ such that $\mathfrak{b} \cap \mathfrak{h}$ has maximal dimension, then we write $\mathcal{Q}(\mathfrak{b})$ for the set of minimal parabolic subgroups containing exp $\mathfrak{b}$. Note that $\mathcal{Q}(\mathfrak{b}) \subseteq \mathcal{Q}$.

COROLLARY 1.10

(i) Let $\mathfrak{a} \in \mathcal{A}$, and let $P \in \mathcal{P}$. Then there exists an element $h \in H$ such that $h P h^{-1} \in \mathcal{P}(\mathfrak{a})$.

(ii) Let $\mathfrak{b}$ be a $\sigma$-stable maximal split abelian subalgebra of $\mathfrak{g}$ such that $\mathfrak{b} \cap \mathfrak{h}$ has maximal dimension. Then for every $Q \in \mathcal{Q}$ there exists an element $h \in H$ such that $h Q h^{-1} \in \mathcal{Q}(\mathfrak{b})$.

\section{Proof}

(i) There exists a $\mathfrak{b} \in \mathcal{A}$ such that $\exp \mathfrak{b} \subseteq P$. By Proposition 1.4 there exists an $h \in H$ such that $A=h B h^{-1}$. This implies that $A$ is contained in $h P h^{-1}$.

(ii) The claim follows from the fact that all such subalgebras are $H$-conjugate. See Lemma 1.1 and [14, Lemmas 4 and 7].

\subsection{Positive systems of $\mathfrak{h}$-roots}

Let $A$ be a $\sigma$-stable connected maximal split abelian subgroup of $G$, and let $\mathfrak{a}$ be its Lie algebra. We write $\Sigma(\mathfrak{a})$ for the root system of $\mathfrak{a}$ in $\mathfrak{g}$. Given a root $\alpha \in \Sigma(\mathfrak{a})$, we write $\mathfrak{g}_{\alpha}$ for the associated root space. 


\section{DEFINITION 1.11}

By an $\mathfrak{h}$-root in $\Sigma(\mathfrak{a})$ we mean a root $\alpha \in \Sigma(\mathfrak{a})$ such that $\mathfrak{g}_{\alpha} \cap \mathfrak{h} \neq\{0\}$. The set of such roots is denoted by $\Sigma_{\mathfrak{h}}(\mathfrak{a})$.

\section{PROPOSITION 1.12}

(i) If $\alpha \in \Sigma_{\mathfrak{h}}(\mathfrak{a})$, then $\left.\alpha\right|_{\mathfrak{a} \cap \mathfrak{q}}=0$.

(ii) $Z_{\mathfrak{h}}(\mathfrak{a} \cap \mathfrak{q})$ is a reductive Lie algebra of which $\mathfrak{a} \cap \mathfrak{h}$ is a maximal split abelian subalgebra. The root system of $\mathfrak{a} \cap \mathfrak{h}$ in $Z_{\mathfrak{h}}(\mathfrak{a} \cap \mathfrak{q})$ equals $\Sigma_{\mathfrak{h}}(\mathfrak{a})$, viewed as a subset of $(\mathfrak{a} \cap \mathfrak{h})^{*}$.

(iii) Assume that $\mathfrak{a} \in \mathcal{A}$. Then $\Sigma_{\mathfrak{h}}(\mathfrak{a})=\Sigma(\mathfrak{a}) \cap(\mathfrak{a} \cap \mathfrak{h})^{*}$. Furthermore, if $\alpha \in$ $\Sigma_{\mathfrak{h}}(\mathfrak{a})$, then $\mathfrak{g}_{\alpha} \subseteq \mathfrak{h}$.

\section{Proof}

(i) If $\mathfrak{g}_{\alpha} \cap \mathfrak{h} \neq\{0\}$, then $\mathfrak{g}_{\sigma \alpha} \cap \mathfrak{g}_{\alpha}=\sigma\left(\mathfrak{g}_{\alpha}\right) \cap \mathfrak{g}_{\alpha} \neq\{0\}$. This implies that $\sigma \alpha=\alpha$.

(ii) Let $\theta$ be a Cartan involution giving rise to a Cartan decomposition as in Lemma 1.1. Since $Z_{\mathfrak{h}}(\mathfrak{a} \cap \mathfrak{q})$ is $\theta$-stable, it follows from [10, Corollary 6.29] that $Z_{\mathfrak{h}}(\mathfrak{a} \cap \mathfrak{q})$ is reductive. The maximality of $\mathfrak{a}$ implies that $\mathfrak{a} \cap \mathfrak{h}$ is a maximal split abelian subalgebra of $Z_{\mathfrak{h}}(\mathfrak{a} \cap \mathfrak{q})$. Let $\Phi$ be the root system of $\mathfrak{a} \cap \mathfrak{h}$ in $Z_{\mathfrak{h}}(\mathfrak{a} \cap \mathfrak{q})$. It follows from (i) that $\left.\alpha\right|_{\mathfrak{a} \cap \mathfrak{h}} \in \Phi$ for every $\alpha \in \Sigma_{\mathfrak{h}}(\mathfrak{a})$. Now let $\beta \in \Phi$, and let $\alpha \in \mathfrak{a}^{*}$ be given by $\left.\alpha\right|_{\mathfrak{a} \cap \mathfrak{h}}=\beta$ and $\left.\alpha\right|_{\mathfrak{a} \cap \mathfrak{q}}=0$. Then $\mathfrak{g}_{\alpha} \cap \mathfrak{h}$ contains the root space of $\beta$; hence, $\alpha \in \Sigma_{\mathfrak{h}}(\mathfrak{a})$. We conclude that $\Phi=\Sigma_{\mathfrak{h}}(\mathfrak{a})$.

(iii) It suffices to prove that, under the given assumption, $\mathfrak{g}_{\alpha} \subseteq \mathfrak{h}$ for every $\alpha \in \Sigma(\mathfrak{a})$ that vanishes on $\mathfrak{a} \cap \mathfrak{q}$. Let $\alpha$ be such a root, and let $X \in \mathfrak{g}_{\alpha} \cap \mathfrak{q}$. We write $\theta$ for a Cartan involution giving rise to a Cartan decomposition as in Lemma 1.1. Since $X$ is in the centralizer of $\mathfrak{a} \cap \mathfrak{q}$, it follows from the maximality of $\mathfrak{a} \cap \mathfrak{q}$ that $X-\theta(X) \in \mathfrak{a} \cap \mathfrak{q}$. Since $X-\theta(X) \in \mathfrak{g}_{\alpha} \oplus \mathfrak{g}_{-\alpha}$, it follows that $X-\theta(X)=0$ and therefore $X=0$.

If $P \in \mathcal{P}(\mathfrak{a})$, then we write $\Sigma(\mathfrak{a} ; P)$ for the positive system of $\Sigma(\mathfrak{a})$ consisting of roots that are positive with respect to $P$. In other words, a root $\alpha \in \Sigma(\mathfrak{a})$ is an element of $\Sigma(\mathfrak{a} ; P)$ if and only if the root space $\mathfrak{g}_{\alpha}$ is contained in $\mathfrak{n}_{P}$.

We fix a positive system $\Sigma_{\mathfrak{h}}^{+}(\mathfrak{a})$ of $\Sigma_{\mathfrak{h}}(\mathfrak{a})$.

\section{COROLLARY 1.13}

Let $Q$ be a minimal parabolic subgroup, and let $A$ be a $\sigma$-stable split component of $Q$. Then $Q$ is $\left(N_{H}(\mathfrak{a} \cap \mathfrak{h}) \cap Z_{H}(\mathfrak{a} \cap \mathfrak{q})\right)$-conjugate to a minimal parabolic subgroup $P$ such that

$$
\Sigma_{\mathfrak{h}}^{+}(\mathfrak{a}) \subseteq \Sigma(\mathfrak{a} ; P)
$$

\section{Proof}

The set $\Sigma_{\mathfrak{h}}(\mathfrak{a}) \cap \Sigma(\mathfrak{a} ; Q)$ is a positive system for the root system $\Sigma_{\mathfrak{h}}(\mathfrak{a})$. It follows from Proposition 1.12(ii) that each such positive system is conjugate to $\Sigma_{\mathfrak{h}}^{+}(\mathfrak{a})$ by an element of the normalizer of $\mathfrak{a} \cap \mathfrak{h}$ in $Z_{H}(\mathfrak{a} \cap \mathfrak{q})$. 


\section{The symmetric space under consideration}

\subsection{The space $X_{n}$}

For the remainder of this article $n$ will be a natural number with $n \geq 3$ and $G$ will be the real Lie group $\operatorname{SL}(n, \mathbb{R})$. Let $\sigma$ be the involution on $G$ given by

$$
\sigma(g)=S g S^{-1}
$$

where

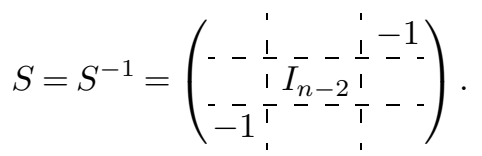

The fixed-point subgroup $H$ of $\sigma$ is obtained from the $\operatorname{subgroup} \operatorname{S}(\operatorname{GL}(n-1, \mathbb{R}) \times$ $\mathrm{GL}(1, \mathbb{R}))$ of $G$ (embedded in the usual manner) by conjugation with the orthogonal matrix

$$
\kappa=\left(\begin{array}{l:l:l}
\frac{1}{\sqrt{2}} & & \frac{1}{\sqrt{2}} \\
\hdashline-I_{n} & I_{n-2} & \\
\hdashline \frac{-1}{\sqrt{2}} & & \frac{1}{\sqrt{2}}
\end{array}\right) .
$$

We denote the $(2 n-2)$-dimensional reductive symmetric space $G / H$ by $X_{n}$.

Let $\theta$ be the Cartan involution given by

$$
\theta(g)=\left(g^{-1}\right)^{t},
$$

and let $K$ be the fixed-point subgroup of $\theta$, that is, $K$ is the maximal compact subgroup $\operatorname{SO}(n)$. Since $S$ is orthogonal, the involutions $\sigma$ and $\theta$ commute. The Lie algebra $\mathfrak{g}$ of $G$ admits the decomposition $\mathfrak{g}=\mathfrak{h} \oplus \mathfrak{q}$ into the eigenspaces for $\sigma$. Here

$$
\mathfrak{h}=\left\{\left(\begin{array}{c:c:c}
-\operatorname{tr} x & v^{t} & y \\
\hdashline-2 & x & -\bar{w} \\
\hdashline y & -v^{t} & \frac{-\operatorname{tr} x}{2}
\end{array}\right): x \in \operatorname{Mat}(n-2, \mathbb{R}), y \in \mathbb{R}, v \in \mathbb{R}^{n-2}, w \in \mathbb{R}^{n-2}\right\}
$$

is the Lie algebra of $H$ and

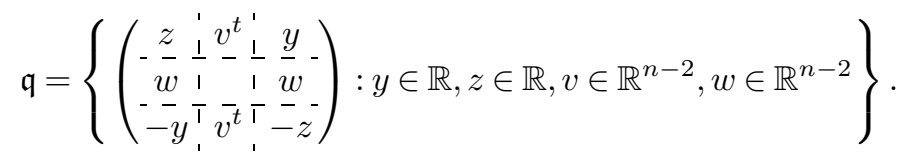

Similarly, we have the Cartan decomposition $\mathfrak{g}=\mathfrak{k} \oplus \mathfrak{p} ;$ here $\mathfrak{k}$ equals $\mathfrak{s o}(n)$ and $\mathfrak{p}$ equals the space of traceless symmetric $n \times n$ matrices. We note that

$$
\mathfrak{a}_{\mathfrak{q}}:=\mathbb{R} \operatorname{diag}(1,0, \ldots, 0,-1)
$$

is a maximal abelian subspace of $\mathfrak{p} \cap \mathfrak{q}$. Hence, the split rank of $X_{n}$ is equal to 1 . We put $A_{\mathfrak{q}}:=\exp \left(\mathfrak{a}_{\mathfrak{q}}\right)$ and for $t \in \mathbb{R}$ we define $a_{t} \in A_{\mathfrak{q}}$ by

$$
a_{t}:=\exp (t \operatorname{diag}(1,0, \ldots, 0,-1)) .
$$




\subsection{Polar decomposition}

The space $X_{n}$ admits a polar decomposition: the map

$$
K \times A_{\mathfrak{q}} \rightarrow X_{n}, \quad(k, a) \mapsto k a \cdot H
$$

is surjective. If $a \cdot H \in K a^{\prime} \cdot H$ for $a, a^{\prime} \in A_{\mathfrak{q}}$, then there exists a $k$ in $N_{K \cap H}\left(\mathfrak{a}_{\mathrm{q}}\right)$, the normalizer of $\mathfrak{a}_{\mathfrak{q}}$ in $K \cap H$, such that $a=k a^{\prime} k^{-1}$. In fact, since the action of $N_{K \cap H}\left(\mathfrak{a}_{\mathrm{q}}\right)$ on $\mathfrak{a}_{\mathfrak{q}}$ is length-preserving (with respect to the Killing form) and

$$
k_{0}=\left(\begin{array}{cc:c:c}
0 & 0 & & 1 \\
0 & -1 & 0 \\
\hdashline-1 & -1 & I_{n-3} & 0 \\
\hdashline 1 & 0 & & 0
\end{array}\right)
$$

is an element in $K \cap H$ such that $k_{0} a k_{0}^{-1}=a^{-1}$ for every $a \in A_{\mathfrak{q}}$, we have $a \cdot H \in$ $K a^{\prime} \cdot H$ if and only if $a^{\prime} \in\left\{a, a^{-1}\right\}$.

LEMMA 2.1

Let $g \in G$ and $t \in \mathbb{R}$. If $g \in K a_{t} \cdot H$, then

$$
\left\|g \sigma(g)^{-1}\right\|_{H S}^{2}=n-2+2 \cosh (4 t),
$$

where $\|\cdot\|_{H S}$ denotes the Hilbert-Schmidt norm on $\operatorname{Mat}(n, \mathbb{R})$.

Proof

A straightforward computation shows that

$$
\left\|g \sigma(g)^{-1}\right\|_{H S}^{2}=\operatorname{tr}\left(g \sigma(g)^{-1}\left(g \sigma(g)^{-1}\right)^{t}\right)=\operatorname{tr}\left(a_{t}^{4}\right) .
$$

The result now follows from the fact that $a_{t}^{4}=\operatorname{diag}\left(e^{4 t}, 1, \ldots, 1, e^{-4 t}\right)$.

\subsection{Schwartz functions}

In this section we give a definition of the space of Schwartz functions on $X_{n}$. This definition differs from the one in [3, Section 17], but it is easily seen, from [3, Theorem 17.1 and Proposition 17.2] combined with Remark 4.5 of the present article, that the definitions are equivalent.

\section{DEFINITION 2.2}

A Schwartz function on $X_{n}$ is a smooth function $\phi: X_{n} \rightarrow \mathbb{C}$ such that for every $u \in U(\mathfrak{g})$ and $m \geq 0$ the seminorm

$$
\sup _{k \in K, t \in \mathbb{R}} \cosh ^{n-1}(t)(1+|t|)^{m}\left|(u \phi)\left(k a_{t} \cdot H\right)\right|
$$

is finite. Here $u \phi$ is obtained with the regular action of $U(\mathfrak{g})$ from the left. The vector space of Schwartz functions on $X_{n}$, equipped with the locally convex topology induced by these seminorms, is denoted by $\mathcal{C}\left(X_{n}\right)$.

\section{REMARK 2.3}

To simplify computational expressions later on, it will be useful to work with the 
following seminorms instead, for $u \in U(\mathfrak{g})$ and $m \in \mathbb{N}$ :

$$
\mu_{u, m}(\phi):=\sup _{k \in K, t \in \mathbb{R}}(2 \cosh 4 t)^{\frac{n-1}{4}}(1+\log (2 \cosh (4 t)))^{m}\left|(u \phi)\left(k a_{t} \cdot H\right)\right| .
$$

Obviously, a smooth function $\phi: X_{n} \rightarrow \mathbb{C}$ belongs to $\mathcal{C}\left(X_{n}\right)$ if and only if these seminorms are finite. Moreover, the seminorms $\mu_{u, m}$ determine the Fréchet topology on $\mathcal{C}\left(X_{n}\right)$.

For future reference we shall now construct some specific Schwartz functions on $X_{n}$.

LEMMA 2.4

Let $\nu<\frac{1-n}{4}$. The function $\phi_{\nu}: X_{n} \rightarrow \mathbb{R}$, defined by

$$
\phi_{\nu}\left(k a_{t} \cdot H\right)=\cosh ^{\nu}(4 t),
$$

is Schwartz.

Proof

We will show that $\mathcal{C}\left(X_{n}\right)$ contains every function $\phi \in C^{\infty}\left(X_{n}\right)$ such that

$$
K \times(1, \infty) \rightarrow \mathbb{C}, \quad(k, t) \mapsto \phi\left(k a_{t} \cdot H\right)
$$

is a linear combination of functions of the form

$$
K \times(1, \infty) \rightarrow \mathbb{C}, \quad(k, t) \mapsto \varphi(k) \cosh ^{\lambda}(t) \sinh ^{\mu}(t),
$$

with $\varphi \in C^{\infty}(K)$ and real numbers $\lambda, \mu$ with sum $\lambda+\mu=4 \nu<1-n$. Clearly our function $\phi_{\nu}$ has this property. It is easily seen that $\mu_{1, m}(\phi)<\infty$ for such a function, and hence, it suffices to show that this class of functions is invariant under the left action by $U(\mathfrak{g})$. For this it suffices to consider the action by $\mathfrak{g}$. Let $X \in \mathfrak{g}$. Then we can write $X$ as a linear combination of elements of the form $\operatorname{Ad}(k) Y$ with some fixed basis elements $Y \in \mathfrak{g}$ and with coefficients which are smooth functions of $k \in K$. We shall use a basis of weight vectors $Y$ for the adjoint action of $\mathfrak{a}_{\mathfrak{q}}$ on $\mathfrak{g}$. If $Y$ belongs to $\mathfrak{a}_{\mathfrak{q}}$ or $Z_{\mathfrak{h}}\left(\mathfrak{a}_{\mathfrak{q}}\right)$, then it is easily seen that $(k, t) \mapsto[\operatorname{Ad}(k)(Y) \phi]\left(k a_{t} \cdot H\right)$ will again be of the form (2.5) with $\lambda+\nu=4 \nu$. It remains to consider the case where $Y$ is a root vector for a root $\alpha$ of $\mathfrak{a}_{\mathfrak{q}}$.

Since $\mathfrak{a}_{\mathfrak{q}}$ is $\sigma \theta$-invariant, the associated root space in $\mathfrak{g}$ is $\sigma \theta$-invariant as well; hence we may assume that $\sigma \theta Y= \pm Y$. If $\sigma \theta Y=Y$, then a simple computation shows that

$$
Y=\frac{-a^{-\alpha}}{a^{\alpha}-a^{-\alpha}}(Y+\theta Y)+\frac{1}{a^{\alpha}-a^{-\alpha}} \operatorname{Ad}(a)(Y+\sigma Y)
$$

for all $a \in A_{\mathfrak{q}}$, and if $\sigma \theta Y=-Y$, then the corresponding formula reads

$$
Y=\frac{a^{-\alpha}}{a^{\alpha}+a^{-\alpha}}(Y+\theta Y)+\frac{1}{a^{\alpha}+a^{-\alpha}} \operatorname{Ad}(a)(Y+\sigma Y) .
$$

Note that, for $a=a_{t}$, in both cases the coefficient of $Y+\theta Y$ is a linear combination of functions $\cosh ^{p} t \sinh ^{q} t$ with $p+q=0$. Hence, when we apply $\operatorname{Ad}(k) Y$ 
to $\phi$ as above, the term with $Y+\theta Y$ will produce a new function of the same kind, whereas the term with $\operatorname{Ad}\left(a_{t}\right)(Y+\sigma Y)$ will be annihilated because of the $H$-invariance from the right. This proves the claim.

\section{4. h-compatible parabolic subgroups}

Let $P$ be a minimal parabolic subgroup. By Theorem 1.8 there exists a $\sigma$-stable split component $A$ of $P$, which is unique up to conjugation by $P \cap H$. We fix such a split component $A$ and write $\mathfrak{a}$ for its Lie algebra. We recall the definition of the root system $\Sigma_{\mathfrak{h}}(\mathfrak{a})$ of $\mathfrak{h}$-roots from Definition 1.11. We write $\Sigma_{\mathfrak{h}}(\mathfrak{a} ; P)$ for the positive system $\Sigma(\mathfrak{a} ; P) \cap \Sigma_{\mathfrak{h}}(\mathfrak{a})$ of $\Sigma_{\mathfrak{h}}(\mathfrak{a})$ and define

$$
\rho_{P, \mathfrak{h}}=\frac{1}{2} \sum_{\alpha \in \Sigma_{\mathfrak{h}}(\mathfrak{a} ; P)} \alpha .
$$

\section{DEFINITION 2.5}

The parabolic subgroup $P$ is said to be $\mathfrak{h}$-compatible if one of the following conditions is fulfilled:

(a) $P$ is of $\sigma$-parabolic rank 1 and $\left\langle\alpha, \rho_{P, \mathfrak{h}}\right\rangle \geq 0$ for all $\alpha \in \Sigma(\mathfrak{a} ; P)$;

(b) $P$ is of $\sigma$-parabolic rank 0 and $\left\langle\alpha, \rho_{P, \mathfrak{h}}\right\rangle>0$ for all $\alpha \in \Sigma(\mathfrak{a} ; P)$.

\section{REMARK 2.6}

We note that this notion is independent of the choice of $\mathfrak{a}$, since any other choice would be $(P \cap H)$-conjugate to $\mathfrak{a}$. Furthermore, it is now readily seen that the property of $\mathfrak{h}$-compatibility is preserved under conjugation by $H$.

Since $X_{n}$ is of split rank 1, every minimal parabolic subgroup is either of $\sigma$ parabolic rank 1 or of $\sigma$-parabolic rank 0 . We recall that $\mathcal{P}$ denotes the set of minimal parabolic subgroups of $\sigma$-parabolic rank 1 and $\mathcal{Q}$ denotes the set of minimal parabolic subgroups of $\sigma$-parabolic rank 0 . We write $\mathcal{P}_{\mathfrak{h}}$ and $\mathcal{Q}_{\mathfrak{h}}$ for the sets of $\mathfrak{h}$-compatible parabolic subgroups in $\mathcal{P}$ and $\mathcal{Q}$, respectively. Recall that $\mathcal{P}_{*}$ denotes the set of $P \in \mathcal{P}$ such that the dimension of $N_{P} /\left(N_{P} \cap H\right)$ is minimal, that is, $\operatorname{dim}\left(N_{P} /\left(N_{P} \cap H\right)\right)=n-1$ (see Proposition 4.4 and (4.10)).

For every $n \geq 3$ the set $\mathcal{P}_{\mathfrak{h}}$ is nonempty. If $n$ is even, then $H$ acts transitively on $\mathcal{P}_{\mathfrak{h}}$ and $\mathcal{P}_{\mathfrak{h}} \subseteq \mathcal{P}_{*}$. If $n$ is odd, then the $H$-action admits three orbits (see Proposition 4.4). One orbit is equal to $\mathcal{P}_{\mathfrak{h}} \backslash\left(\mathcal{P}_{*} \cap \mathcal{P}_{\mathfrak{h}}\right)$; the other two orbits are contained in $\mathcal{P}_{*} \cap \mathcal{P}_{\mathfrak{h}}$.

If $n$ is even, then $\mathcal{Q}_{\mathfrak{h}}=\emptyset$. If $n$ is odd, then $\mathcal{Q}_{\mathfrak{h}}$ is nonempty and $H$ acts transitively on it (see Proposition 5.3).

\section{Main theorems}

\subsection{Convergence}

Let $P$ be a minimal parabolic subgroup of $G$, and let $d x$ be a nonzero $N_{P^{-}}$ invariant Radon measure on $N_{P} /\left(N_{P} \cap H\right)$. 


\section{THEOREM 3.1}

The integral

$$
\int_{N_{P} /\left(N_{P} \cap H\right)} \phi(x \cdot H) d x
$$

is absolutely convergent for every $\phi \in \mathcal{C}\left(X_{n}\right)$ if and only if $P$ is $\mathfrak{h}$-compatible.

The proof of this theorem will be given in Sections 4 and 5 .

\subsection{Limit behavior}

Assume that $P \in \mathcal{P}_{\mathfrak{h}} \cap \mathcal{P}_{*}$. Let $A$ be a $\sigma$-stable split component of $P$, and let $L$ be the centralizer of $A$ in $G$. Then $L=M A$, where $M$ is the (unique) maximal compact subgroup of $L$. Now $P=L N_{P}$ and $P=M A N_{P}$ are a Levi and a Langlands decomposition, respectively. Note that $\mathfrak{a}=\operatorname{Lie}(A) \in \mathcal{A}$. We define

$$
\delta_{P}: L \rightarrow \mathbb{R}_{>0}, \quad l \mapsto\left|\frac{\left.\operatorname{det} \operatorname{Ad}(l)\right|_{\mathfrak{n}_{P}}}{\left.\operatorname{det} \operatorname{Ad}(l)\right|_{\mathfrak{n}_{P} \cap Z_{\mathfrak{g}}(\mathfrak{a} \cap \mathfrak{q})}}\right|^{\frac{1}{2}} .
$$

Let

$$
\rho_{P}=\frac{1}{2} \sum_{\alpha \in \Sigma(\mathfrak{a} ; P)} \alpha
$$

Then for $m \in M$ and $a \in A$

$$
\delta_{P}(m a)=a^{\rho_{P}-\rho_{P, \mathfrak{h}}} .
$$

For $\phi \in \mathcal{C}\left(X_{n}\right)$ we define its Harish-Chandra transform $\mathcal{H}_{P} \phi$ to be the function on $L /(L \cap H)$ given by

$$
\mathcal{H}_{P} \phi(l)=\delta_{P}(l) \int_{N_{P} /\left(N_{P} \cap H\right)} \phi(l n) d n \quad(l \in L) .
$$

Note that the integrals are absolutely convergent by Theorem 3.1 and define a right $(L \cap H)$-invariant function on $L$.

\section{THEOREM 3.2}

Let $P \in \mathcal{P}_{\mathfrak{h}} \cap \mathcal{P}_{*}$ be as above, and let $v \in \mathfrak{a} \cap \mathfrak{q}$ be such that $\rho_{P}(v)>0$.

(i) Assume that $n$ is even. Then for every $\phi \in \mathcal{C}\left(X_{n}\right)$ and every $N \in \mathbb{N}$,

$$
\sup _{m \in M, t \in \mathbb{R}}\left|t^{N} \mathcal{H}_{P} \phi(m \exp (t v))\right|<\infty .
$$

(ii) Assume that $n$ is odd. Then for every $\phi \in \mathcal{C}\left(X_{n}\right)$, every $r \in \mathbb{R}$, and every $N \in \mathbb{N}$,

$$
\sup _{m \in M, t<r}\left|t^{N} \mathcal{H}_{P} \phi(m \exp (t v))\right|<\infty .
$$

Moreover, the limit

$$
\mu_{P}(\phi):=\lim _{t \rightarrow \infty} \mathcal{H}_{P} \phi(\exp (t v))
$$


exists, and there exist an $\mathfrak{h}$-compatible $Q \in \mathcal{Q}$ and an element $g \in G$ such that, for every $\phi \in \mathcal{C}\left(X_{n}\right)$,

$$
\mu_{P}(\phi)=\int_{N_{Q} /\left(N_{Q} \cap H\right)} \phi(g n) d n .
$$

Vice versa, if $Q \in \mathcal{Q}$, then there exist a $P^{\prime} \in \mathcal{P}_{\mathfrak{h}} \cap \mathcal{P}_{*}$ and an element $g \in G$ such that, for every $\phi \in \mathcal{C}\left(X_{n}\right)$,

$$
\int_{N_{Q} /\left(N_{Q} \cap H\right)} \phi(g n) d n=\mu_{P^{\prime}}(\phi) .
$$

The proof of the theorem will be given in Section 6 .

\subsection{Cusp forms}

As explained in the Introduction, the aim of the article is to explore which parabolic subgroups should be used in the definition of cusp forms for reductive symmetric spaces of split rank 1 . In [4] it was proved that for such a symmetric space $X$ the integral

$$
\int_{N_{P} /\left(N_{P} \cap H\right)} \phi(n) d n
$$

is absolutely convergent for all $\phi \in \mathcal{C}(X)$ and every $P \in \mathcal{P}_{\mathfrak{h}}$.

It follows from Theorem 3.1 that for the spaces $X_{n}$ only the $\mathfrak{h}$-compatible parabolic subgroups provide integrals that are convergent for all Schwartz functions. We conclude from this that the condition that $P$ is $\mathfrak{h}$-compatible, which was needed in [4], is not an artifact of the proof.

For $n$ odd the set $\mathcal{Q}_{\mathfrak{h}}$ is nonempty. In [4] only the minimal parabolic subgroups from $\mathcal{P}_{\mathfrak{h}}$ were used. The remaining question that needs to be answered is whether the class of parabolic subgroups that is used for the definition of cusp forms should include any minimal parabolic subgroup from $\mathcal{Q}_{\mathfrak{h}}$.

\section{PROPOSITION 3.3}

Let $\phi \in \mathcal{C}\left(X_{n}\right)$. The following four conditions are equivalent.

(i) For every $g \in G$ and every $\mathfrak{h}$-compatible parabolic subgroup $P$,

$$
\int_{N_{P} /\left(N_{P} \cap H\right)} \phi(g n) d n=0 .
$$

(ii) For every $g \in G$ and every $P \in \mathcal{P}_{\mathfrak{h}}$, (3.1) holds.

(iii) For every $g \in G$ and every $P \in \mathcal{P}_{\mathfrak{h}} \cap \mathcal{P}_{*}$, (3.1) holds.

(iv) There exists a $P$ in each $H$-conjugacy class in $\mathcal{P}_{\mathfrak{h}} \cap \mathcal{P}_{*}$ such that, for every $g \in G$, (3.1) holds.

Proof

The implications (i) $\Rightarrow$ (ii) $\Rightarrow$ (iii) $\Rightarrow$ (iv) are trivial. 
Conversely, if (3.1) holds for a given $\mathfrak{h}$-compatible parabolic subgroup $P$ and every $g \in G$, then it also holds for every $H$-conjugate of $P$. This proves (iv) $\Rightarrow$ (iii). The implication (iii) $\Rightarrow$ (ii) is proved in [4, Lemma 8.14].

Now assume $Q \in \mathcal{Q}_{\mathfrak{h}}$. By Theorem 3.2 the integrals over $N_{Q} /\left(N_{Q} \cap H\right)$ can be obtained as limits of integrals over $N_{P} /\left(N_{P} \cap H\right)$ for some $P \in \mathcal{P}_{\mathfrak{h}} \cap \mathcal{P}_{*}$. This establishes the implication (ii) $\Rightarrow$ (i).

\section{DEFINITION 3.4}

A function $\phi \in \mathcal{C}\left(X_{n}\right)$ is said to be a cusp form if one of the equivalent conditions in Proposition 3.3 is satisfied.

We write $\mathcal{C}_{\text {cusp }}\left(X_{n}\right)$ for the space of cusp forms on $X_{n}$ and $\mathcal{C}_{\mathrm{ds}}\left(X_{n}\right)$ for the closed span of the discrete series representations of $X_{n}$. Given $g \in G$ and $\phi \in \mathcal{C}\left(X_{n}\right)$, we write $L_{g} \phi$ for the function given by $L_{g} \phi(x)=\phi\left(g^{-1} x\right)$, for $x \in X_{n}$. In [4] the following theorem, which we here only state for $X_{n}$, is proved for general reductive symmetric spaces of split rank 1 .

\section{THEOREM 3.5}

(i) $\mathcal{C}_{\text {cusp }}\left(X_{n}\right) \subseteq \mathcal{C}_{\mathrm{ds}}\left(X_{n}\right)$.

(ii) If $\mathcal{C}_{\mathrm{ds}}\left(X_{n}\right)^{K} \subseteq \mathcal{C}_{\text {cusp }}\left(X_{n}\right)^{K}$, then $\mathcal{C}_{\mathrm{ds}}\left(X_{n}\right)=\mathcal{C}_{\text {cusp }}\left(X_{n}\right)$.

(iii) Fix $\mathfrak{a} \in \mathcal{A}$ with $\mathfrak{a}_{\mathfrak{q}} \subseteq \mathfrak{a}$. Let $\phi \in \mathcal{C}\left(X_{n}\right)$ be $K$-finite. Then $\phi \in \mathcal{C}_{\mathrm{ds}}\left(X_{n}\right)$ if and only if for every $\mathfrak{h}$-compatible minimal parabolic $P \in \mathcal{P}(\mathfrak{a})$ and every $k \in K$ the function

$$
\left.\mathcal{H}_{P}\left(L_{k} \phi\right)\right|_{A_{\mathfrak{q}}}
$$

is a finite linear combination of exponential functions with nonzero exponents.

Theorem 3.5 and the estimates in Theorem 3.2 have the following corollary.

\section{THEOREM 3.6}

We have that $\mathcal{C}_{\mathrm{ds}}\left(X_{n}\right)=\mathcal{C}_{\text {cusp }}\left(X_{n}\right)$.

\section{Proof}

By Theorem 3.5(i) and (ii) it suffices to prove that $\mathcal{C}_{\mathrm{ds}}\left(X_{n}\right)^{K} \subseteq \mathcal{C}_{\text {cusp }}\left(X_{n}\right)^{K}$. Let $\phi \in \mathcal{C}_{\mathrm{ds}}\left(X_{n}\right)^{K}$. We need to show that $\phi$ is a cusp form. For this we will prove that condition (3.3) in Proposition 3.3 is satisfied.

By Theorem 3.5(iii) the restriction of $\mathcal{H}_{P} \phi$ to $A_{\mathfrak{q}}$ is of exponential type with nonzero exponents. From Theorem 3.2 it follows that this function is bounded. The only function on $A_{\mathfrak{q}}$ that satisfies both conditions is the 0 -function. This proves that

$$
\int_{N_{P} /\left(N_{P} \cap H\right)} \phi(a n) d n=0
$$

for every $a \in A_{\mathfrak{q}}$. Now, let $g \in G$. By the Iwasawa decomposition there exist $k \in K$, $a \in A_{\mathfrak{q}}$, and $a_{H} \in A \cap H$ such that $g \in k a a_{H} N_{P}$. Using that $\phi$ is $K$-invariant, we 
find

$$
a_{H}^{\rho_{P}-\rho_{P, \mathfrak{h}}} \int_{N_{P} /\left(N_{P} \cap H\right)} \phi(g n) d n=\int_{N_{P} /\left(N_{P} \cap H\right)} \phi(a n) d n=0 .
$$

This proves the claim.

\section{Proof of Theorem 3.1 for $\sigma$-parabolic rank 1}

In this section we deal with the proof for Theorem 3.1 under the assumption that $P$ is of $\sigma$-parabolic rank 1. In Sections $4.1-4.4$ we first reduce the statement to a (seemingly) less general statement, which we then prove in Sections 4.5-4.7.

\subsection{Root systems}

We recall the definitions of $\mathfrak{a}_{\mathfrak{q}}, A_{\mathfrak{q}}$, and $a_{t}$ from Section 2.1 , and define

$$
\mathfrak{a}_{\mathfrak{h}}:=\left\{\operatorname{diag}\left(x_{1}, x_{2}, \ldots, x_{n-1}, x_{1}\right): x_{i} \in \mathbb{R}, 2 x_{1}+\sum_{i=2}^{n-1} x_{i}=0\right\} .
$$

Note that $\mathfrak{a}_{\mathfrak{q}}$ is a maximal abelian subspace of $\mathfrak{p} \cap \mathfrak{q}$ and $\mathfrak{a}_{\mathfrak{h}}$ is a subspace of $\mathfrak{p} \cap \mathfrak{h}$ such that $\mathfrak{a}=\mathfrak{a}_{\mathfrak{h}} \oplus \mathfrak{a}_{\mathfrak{q}}$ is a maximal abelian subspace of $\mathfrak{p}$. We write $A$ for $\exp (\mathfrak{a})$.

In the remainder of this section we shall describe the root system of $\mathfrak{a}$ in $\mathfrak{g}$. For $1 \leq k \leq n$ we define the functional

$$
e_{k}: \mathfrak{a} \rightarrow \mathbb{R}, \quad \operatorname{diag}\left(x_{1}, \ldots, x_{n}\right) \mapsto x_{k} .
$$

The root system of $\mathfrak{a}$ in $\mathfrak{g}$ then equals

$$
\Sigma:=\Sigma(\mathfrak{g}, \mathfrak{a})=\left\{e_{i}-e_{j}: 1 \leq i, j \leq n, i \neq j\right\} .
$$

The root spaces are $\mathfrak{g}_{e_{i}-e_{j}}=\mathbb{R} E_{i, j}$, where $E_{i, j}$ is the matrix whose entry on the $i$ th row and $j$ th column equals 1 , whereas all remaining entries equal zero.

Note that

$$
\Sigma_{\mathfrak{h}}:=\Sigma_{\mathfrak{h}}(\mathfrak{a})=\left\{e_{i}-e_{j}: 2 \leq i, j \leq n-1, i \neq j\right\}
$$

is the root system in $\mathfrak{a}_{\mathfrak{h}}$ for $Z_{\mathfrak{h}}\left(\mathfrak{a}_{\mathfrak{q}}\right)$ (see Proposition 1.12(ii)). We set

$$
\Sigma_{\mathfrak{h}}^{+}=\Sigma_{\mathfrak{h}}^{+}(\mathfrak{a})=\left\{e_{i}-e_{j}: 2 \leq i<j \leq n-1\right\}
$$

and define

$$
\rho_{\mathfrak{h}}=\frac{1}{2} \sum_{\alpha \in \Sigma_{\mathfrak{h}}^{+}} \alpha
$$

The set

$$
\Sigma_{\mathfrak{q}}=\Sigma \cap \mathfrak{a}_{\mathfrak{q}}^{*}=\left\{ \pm\left(e_{1}-e_{n}\right)\right\}
$$

forms a root system. We denote the associated positive system $\left\{e_{1}-e_{n}\right\}$ by $\Sigma_{\mathfrak{q}}^{+}$.

\subsection{Classification of parabolic subgroups}

Recall that $\mathcal{P}$ denotes the set of minimal parabolic subgroups containing a $\sigma$ stable maximal connected split abelian subgroup $B$ such that $\mathfrak{b} \cap \mathfrak{q}$ has maximal 
dimension, that is, $\operatorname{dim}(\mathfrak{b} \cap \mathfrak{q})=1$. Recall also that $\mathcal{P}(\mathfrak{a})$ denotes the subset of $\mathcal{P}$ consisting of minimal parabolic subgroups containing $A$. From now on we shall write $\Sigma(P):=\Sigma(\mathfrak{a} ; P)$ for $P \in \mathcal{P}(\mathfrak{a})$.

\section{LEMMA 4.1}

Let $Q \in \mathcal{P}$. Then there exists a parabolic subgroup $P \in \mathcal{P}(\mathfrak{a})$ which is $H$-conjugate to $Q$ and satisfies

$$
\left(\Sigma_{\mathfrak{h}}^{+} \cup \Sigma_{\mathfrak{q}}^{+}\right) \subseteq \Sigma(P) .
$$

Let $P$ be any such parabolic subgroup. Then $\rho_{P, \mathfrak{h}}=\rho_{\mathfrak{h}}$. Moreover, $Q$ is $\mathfrak{h}$ compatible if and only if $P$ is $\mathfrak{h}$-compatible (see Definition 2.5).

Proof

By Corollaries 1.10 and 1.13, $Q$ is $H$-conjugate to a parabolic subgroup $P^{\prime} \in \mathcal{P}(\mathfrak{a})$ with $\Sigma_{\mathfrak{h}}^{+} \subseteq \Sigma\left(P^{\prime}\right)$. If $\Sigma\left(P^{\prime}\right) \cap \mathfrak{a}_{\mathfrak{q}}^{*}=\Sigma_{\mathfrak{q}}^{+}$, then (4.2) holds with $P=P^{\prime}$. Otherwise, recall the element $k_{0}$ from (2.3). This element is in $N_{K \cap H}\left(\mathfrak{a}_{\mathfrak{q}}\right) \cap Z_{K \cap H}\left(\mathfrak{a}_{\mathfrak{h}}\right)$ and acts by inversion on $\mathfrak{a}_{\mathfrak{q}}$. Hence, $P=k_{0} P^{\prime} k_{0}^{-1}$ satisfies (4.2). In particular, $\rho_{P, \mathfrak{h}}=$ $\rho_{\mathfrak{h}}$. The final assertion follows from Remark 2.6.

We will now classify the parabolic subgroups $P \in \mathcal{P}(\mathfrak{a})$ satisfying (4.2). Every parabolic subgroup $P \in \mathcal{P}(\mathfrak{a})$ is uniquely determined by the corresponding positive system $\Sigma(P)$. The set of these positive systems is in bijection with the symmetric group $S_{n}$ : an element $\tau \in S_{n}$ corresponds to the positive system $\Sigma(P)$ given by

$$
\Sigma(P)=\left\{e_{\tau^{-1}(i)}-e_{\tau^{-1}(j)}: 1 \leq i<j \leq n\right\} .
$$

Equivalently, a root $e_{i}-e_{j} \in \Sigma$ belongs to $\Sigma(P)$ if and only if $\tau(i)<\tau(j)$. We thus see that the parabolic subgroups $P \in \mathcal{P}(\mathfrak{a})$ satisfying (4.2) correspond to the $\tau \in S_{n}$ such that

$$
\tau(i)<\tau(j) \quad \text { for } 2 \leq i<j \leq n-1 \quad \text { and } \quad \tau(1)<\tau(n) .
$$

Given such a permutation $\tau \in S_{n}$, there exist a unique $k$, with $2 \leq k \leq n$, such that

$$
\tau(k-1) \leq \tau(1)<\tau(k),
$$

and a unique $l$, with $k \leq l \leq n$, such that

$$
\tau(l-1)<\tau(n) \leq \tau(l) .
$$

Conversely, for each pair of integers $(k, l)$ satisfying $2 \leq k \leq l \leq n$, there exists a unique $\tau \in S_{n}$ satisfying (4.3), (4.4), and (4.5). From now on we write $P_{k, l}$ for the corresponding minimal parabolic subgroup and $\Sigma_{k, l}^{+}$for $\Sigma\left(P_{k, l}\right)$. Furthermore, we write $P_{k, l}=M A N_{k, l}$ for the Langlands decomposition of $P_{k, l}$. For future reference we note that the positive system corresponding to $(k, l)$ is given by the disjoint 
union

$$
\begin{aligned}
\Sigma_{k, l}^{+}= & \Sigma_{\mathfrak{h}}^{+} \cup \Sigma_{\mathfrak{q}}^{+} \cup\left\{e_{j}-e_{1}: 2 \leq j \leq k-1\right\} \cup\left\{e_{1}-e_{j}: k \leq j \leq n-1\right\} \\
& \cup\left\{e_{j}-e_{n}: 2 \leq j \leq l-1\right\} \cup\left\{e_{n}-e_{j}: l \leq j \leq n-1\right\}
\end{aligned}
$$

In the following we assume that $b$ is the ad-invariant bilinear form on $\mathfrak{s l}(n, \mathbb{R})$ given by $b(X, Y)=\operatorname{tr}(X Y)$. It is well known that $b$ is a positive multiple of the Killing form $B$; in fact, $b=\frac{1}{4 n} B$. The restriction of $b$ to $\mathfrak{a}$, denoted $\langle\cdot, \cdot\rangle$, is a positive definite inner product. We equip $\mathfrak{a}^{*}$ with the dual inner product, also denoted $\langle\cdot, \cdot\rangle$, which on two elements $\mu, \nu \in \mathfrak{a}^{*}$ is given as follows. The elements have unique expressions $\mu=\sum_{j} \mu_{j} e_{j}$ and $\nu=\sum_{j} \nu_{j} e_{j}$ provided we demand that $\sum_{j} \mu_{j}=0$ and $\sum_{j} \nu_{j}=0$. In terms of these expressions,

$$
\langle\mu, \nu\rangle=\sum_{j} \mu_{j} \nu_{j}
$$

In particular, each root $e_{i}-e_{j}$ has length $\sqrt{2}$.

\section{LEMMA 4.2}

Let $i$ and $j$ be integers such that $1 \leq i<j \leq n$. Then

$$
\left\langle e_{i}-e_{j}, \rho_{\mathfrak{h}}\right\rangle= \begin{cases}j-i & \text { if } 2 \leq i<j \leq n-1, \\ j-\frac{n+1}{2} & \text { if } i=1 \text { and } 2 \leq j \leq n-1, \\ \frac{n+1}{2}-i & \text { if } 2 \leq i \leq n-1 \text { and } j=n, \\ 0 & \text { if } i=1 \text { and } j=n .\end{cases}
$$

In particular, $\left\langle\alpha, \rho_{\mathfrak{h}}\right\rangle \geq 0$ for every $\alpha \in \Sigma\left(P_{k, l}\right)$ if and only if $\frac{n+1}{2} \leq k \leq l \leq \frac{n+3}{2}$.

\section{Proof}

Using the definition of $\rho_{\mathfrak{h}}$, we find

$$
2 \rho_{\mathfrak{h}}=\sum_{2 \leq i<j \leq n-1}\left(e_{i}-e_{j}\right)=\sum_{i=2}^{n-1}(n+1-2 i) e_{i} .
$$

The first statement in the lemma is a direct consequence of this formula; the second follows from comparison with (4.6).

\section{LEMMA 4.3}

Let $2 \leq k \leq l \leq n$, and let $\mathfrak{n}_{k, l}$ be the Lie algebra of $N_{k, l}$. Then

$$
\mathfrak{n}_{k, l} \cap \mathfrak{h}=\bigoplus_{\alpha \in \Sigma_{\mathfrak{h}}^{+}} \mathfrak{g}_{\alpha} \oplus \bigoplus_{2 \leq j \leq k-1}(1+\sigma) \mathfrak{g}_{e_{j}-e_{1}} \oplus \bigoplus_{l \leq j \leq n-1}(1+\sigma) \mathfrak{g}_{e_{n}-e_{j}}
$$

Proof

Since $\sigma\left(e_{1}\right)=e_{n}$ and $\sigma\left(e_{j}\right)=e_{j}$ for $2 \leq j \leq n-1$, we find

$$
\begin{aligned}
\Sigma_{k, l}^{+} \cap \sigma\left(\Sigma_{k, l}^{+}\right)= & \Sigma_{\mathfrak{h}}^{+} \cup\left\{e_{j}-e_{1}: 2 \leq j \leq k-1\right\} \cup\left\{e_{1}-e_{j}: l \leq j \leq n-1\right\} \\
& \cup\left\{e_{j}-e_{n}: 2 \leq j \leq k-1\right\} \cup\left\{e_{n}-e_{j}: l \leq j \leq n-1\right\},
\end{aligned}
$$


the union being disjoint. The root spaces $\mathfrak{g}_{\alpha}$ for $\alpha \in \Sigma_{\mathfrak{h}}^{+}$are contained in $\mathfrak{h}$. Furthermore, if both $\alpha$ and $\sigma(\alpha)$ are roots in $\Sigma_{k, l}^{+} \backslash \Sigma_{\mathfrak{h}}^{+}$, then

$$
\mathfrak{g}_{\alpha} \oplus \mathfrak{g}_{\sigma(\alpha)}=(1+\sigma) \mathfrak{g}_{\alpha} \oplus(1-\sigma) \mathfrak{g}_{\alpha}
$$

where the first term on the right-hand side is a subspace of $\mathfrak{n}_{k, l} \cap \mathfrak{h}$ and the second term is a subspace in $\mathfrak{n}_{k, l} \cap \mathfrak{q}$. This proves that the right-hand side of (4.8) is contained in $\mathfrak{n}_{k, l} \cap \mathfrak{h}$.

To prove the converse, assume that $Y \in \mathfrak{n}_{k, l} \cap \mathfrak{h}$. Then $Y=\sum_{\alpha \in \Sigma_{k, l}^{+}} Y_{\alpha}$, where $Y_{\alpha} \in \mathfrak{g}_{\alpha}$. Since $Y \in \mathfrak{h}$, we have $\sigma(Y)=Y$. This implies that $\sigma\left(Y_{\alpha}\right)=Y_{\sigma(\alpha)}$ if both $\alpha$ and $\sigma(\alpha)$ are elements of $\Sigma_{k, l}^{+}$and $Y_{\alpha}=0$ otherwise. Let $\alpha$ be a root such that $Y_{\alpha} \neq 0$. If $\alpha=\sigma(\alpha)$, then $\alpha \in \Sigma_{\mathfrak{h}}^{+}$. If $\alpha \neq \sigma(\alpha)$, then there exists a $2 \leq j \leq k-1$ such that either $\alpha=e_{j}-e_{1}$ or $\sigma(\alpha)=e_{j}-e_{1}$, or there exists a $l \leq j \leq n-1$ such that either $\alpha=e_{n}-e_{j}$ or $\sigma(\alpha)=e_{n}-e_{j}$. Therefore, $Y$ is contained in the right-hand side of (4.8). This proves the lemma.

\section{PROPOSITION 4.4}

Let $P \in \mathcal{P}$. Then there exist unique integers $k$ and $l$, with $2 \leq k \leq l \leq n$, such that $P$ is $H$-conjugate to $P_{k, l}$. Moreover, $P \in \mathcal{P}_{\mathfrak{h}}$ if and only if

$$
\frac{n+1}{2} \leq k \leq l \leq \frac{n+3}{2}
$$

and $P \in \mathcal{P}_{*}$ if and only if $k=l$.

Proof

The existence of the integers $k$ and $l$ follows directly from Lemma 4.1. To prove the uniqueness of these integers, let $2 \leq k \leq l \leq n$, let $2 \leq k^{\prime} \leq l^{\prime} \leq n$, and assume that there exists an $h \in H$ with $h P_{k, l} h^{-1}=P_{k^{\prime}, l^{\prime}}$. Note that $A$ and $h A h^{-1}$ are both $\sigma$-stable split components of $P_{k^{\prime}, l^{\prime}}$. From Theorem 1.8 it follows that there exists a unique $n \in N_{k^{\prime}, l^{\prime}} \cap H$ such that $n h A h^{-1} n^{-1}=A$. We write $h^{\prime}$ for $n h$. Note that $h^{\prime} \in N_{H}(\mathfrak{a})=N_{H}\left(\mathfrak{a}_{\mathfrak{q}}\right) \cap N_{H}\left(\mathfrak{a}_{\mathfrak{h}}\right)$ and $h^{\prime} P_{k, l} h^{\prime-1}=P_{k^{\prime}, l^{\prime}}$. It follows that $h^{\prime}$ acts trivially on $\Sigma_{\mathfrak{q}}^{+}$and therefore $h^{\prime} \in Z_{H}\left(\mathfrak{a}_{\mathfrak{q}}\right)$. From Corollary 1.12 we see that $\Sigma_{\mathfrak{h}}$ is the root system of $\mathfrak{a}_{\mathfrak{h}}$ in $Z_{\mathfrak{h}}\left(\mathfrak{a}_{\mathfrak{q}}\right)$. Since $h^{\prime} \in N_{H}\left(\mathfrak{a}_{\mathfrak{h}}\right) \cap Z_{H}\left(\mathfrak{a}_{\mathfrak{q}}\right)$, it induces an element $w$ in the Weyl group of this root system. As the positive system $\Sigma_{\mathfrak{h}}^{+}$ is contained in both $\Sigma_{k, l}^{+}$and $\Sigma_{k^{\prime}, l^{\prime}}^{+}$, it follows that $w$ acts trivially on $\Sigma_{\mathfrak{h}}^{+}$, and thus we conclude that $h^{\prime}$ acts trivially on $\mathfrak{a}_{\mathfrak{h}}$. This proves that $h^{\prime} P_{k, l} h^{\prime-1}=P_{k, l}$ and hence $k=k^{\prime}$ and $l=l^{\prime}$.

From Lemma 4.2 it is easily seen that $P \in \mathcal{P}_{\mathfrak{h}}$ if and only if $\frac{n+1}{2} \leq k \leq l \leq \frac{n+3}{2}$. The final claim, that $P \in \mathcal{P}_{*}$ if and only if $k=l$, follows from Lemma 4.3.

\section{REMARK 4.5}

We recall from [4, Definition 1.1] that a parabolic subgroup $P \in \mathcal{P}(\mathfrak{a})$ is said to be $\mathfrak{q}$-extreme if $\Sigma(P) \cap \sigma \theta \Sigma(P)=\Sigma(P) \backslash \Sigma_{\mathfrak{h}}$. Since $\sigma \theta e_{j}=-e_{j}$ for $2 \leq j \leq n-1$, whereas $\sigma \theta e_{1}=-e_{n}$, it follows from the characterization of $\Sigma_{k, l}$ above that $P_{k, l}$ is $\mathfrak{q}$-extreme if and only if $k=2$ and $l=n-1$. Let $\Sigma_{0}^{+}$be the positive system for the root system $\Sigma\left(\mathfrak{g}, \mathfrak{a}_{\mathrm{q}}\right)$ obtained by restricting the roots from $\Sigma_{2, n-1} \backslash \Sigma_{\mathfrak{h}}$ to 
$\mathfrak{a}_{\mathrm{q}}$. Then $\Sigma_{0}^{+}=\{\alpha, 2 \alpha\}$, where $2 \alpha=e_{1}-e_{n}$. It is now readily checked that $\alpha$ has multiplicity $2(n-2)$, whereas $2 \alpha$ has multiplicity 1 . Accordingly, $\rho_{0}:=\rho\left(\Sigma_{0}^{+}\right)$is given by $\rho_{0}=(n-1) \alpha$, so that

$$
a_{t}^{\rho_{0}}=e^{(n-1) t} \quad(t \in \mathbb{R}) .
$$

The element $\rho_{0} \in \mathfrak{a}_{\mathrm{q}}^{*}$ defined above corresponds to the element $\rho_{Q}$ appearing in [3, Proposition 17.2]. Accordingly, it follows that Definition 2.2 of the Schwartz space $\mathcal{C}\left(X_{n}\right)$ is consistent with the characterization given in [3, Theorem 17.1].

\subsection{Decomposition and invariant measures}

Let $k$ and $l$ be integers such that $2 \leq k \leq l \leq n$. We recall that $P_{k, l}=Z_{K}(\mathfrak{a}) A N_{k, l}$ is the minimal parabolic subgroup containing $A$ such that $\Sigma\left(P_{k, l}\right)=\Sigma_{k, l}^{+}$, and that the latter root system is given by (4.6).

We define

$$
\mathfrak{u}_{k, l}=\mathfrak{g}_{e_{1}-e_{n}} \oplus \bigoplus_{2 \leq j \leq l-1} \mathfrak{g}_{e_{j}-e_{n}} \oplus \bigoplus_{k \leq j \leq n-1} \mathfrak{g}_{e_{1}-e_{j}}
$$

Note that $\mathfrak{u}_{k, l}$ is a Lie subalgebra of $\mathfrak{n}_{k, l}$. We write $U_{k, l}$ for the Lie subgroup $\exp \left(\mathfrak{u}_{k, l}\right)$. For $x, y \in \mathbb{R}^{n-2}$ and $z \in \mathbb{R}$, we define

$$
u_{x, y, z}=\left(\begin{array}{c:c:c}
1 & x^{t} & z \\
\hdashline & I_{n-2} & 1 \\
\hdashline & - & 1 \\
\hdashline & & 1
\end{array}\right)
$$

A straightforward computation shows that

$$
U_{k, l}=\left\{u_{x, y, z}: x \in\{0\}^{k-2} \times \mathbb{R}^{n-k}, y \in \mathbb{R}^{l-2} \times\{0\}^{n-l}, z \in \mathbb{R}\right\} .
$$

By Lemma 4.3, we have

$$
\mathfrak{n}_{k, l}=\left(\mathfrak{n}_{k, l} \cap \mathfrak{h}\right) \oplus \mathfrak{u}_{k, l} .
$$

LEMMA 4.6

The map

$$
U_{k, l} \times\left(N_{k, l} \cap H\right) \rightarrow N_{k, l}, \quad(u, n) \mapsto u n
$$

is a diffeomorphism. There exist normalizations for the invariant measure $d x$ on $N_{k, l} / N_{k, l} \cap H$ and the Haar measure $d u$ of $U_{k, l}$ such that, for every $\phi \in$ $C_{c}\left(N_{k, l} /\left(N_{k, l} \cap H\right)\right)$,

$$
\int_{N_{k, l} /\left(N_{k, l} \cap H\right)} \phi(x) d x=\int_{U_{k, l}} \phi\left(u \cdot\left(N_{k, l} \cap H\right)\right) d u .
$$

Finally, the normalizations may be chosen such that, in addition, the above integrals equal

$$
\int_{z \in \mathbb{R}} \int_{y \in \mathbb{R}^{l-2} \times\{0\}^{n-l}} \int_{x \in\{0\}^{k-2} \times \mathbb{R}^{n-k}} \phi\left(u_{x, y, z} \cdot\left(N_{k, l} \cap H\right)\right) d x d y d z .
$$




\section{Proof}

The first two assertions follow from [2, Proposition 2.16]. For the final assertion, we note that

$$
u_{x, y, z+\langle x, y\rangle / 2}=\exp \left(\begin{array}{c:c:c}
0 & x^{t} & z \\
\hdashline 1 & 0 & 1 \\
\hdashline & 0,-2 & y \\
\hdashline & - & 0
\end{array}\right) .
$$

It follows that, up to a suitable normalization of measures, the second integral in (4.11) equals

$$
\int_{z \in \mathbb{R}} \int_{y \in \mathbb{R}^{l-2} \times\{0\}^{n-l}} \int_{x \in\{0\}^{k-2} \times \mathbb{R}^{n-k}} \phi\left(u_{x, y, z+\langle x, y\rangle / 2} \cdot\left(N_{k, l} \cap H\right)\right) d x d y d z .
$$

The equality with (4.12) now follows from a simple substitution of variables.

To conclude this section we state one more lemma.

\section{LEMMA 4.7}

Let $w$ be the longest Weyl group element for $\Sigma_{\mathfrak{h}}=\Sigma\left(Z_{\mathfrak{h}}\left(\mathfrak{a}_{\mathfrak{q}}\right) ; \mathfrak{a}_{\mathfrak{h}}\right)$, relative to the positive system (4.1), and let $w_{0} \in N_{K \cap H}(\mathfrak{a}) \cap Z_{K \cap H}\left(\mathfrak{a}_{\mathfrak{q}}\right)$ be a representative for w. Then

$$
\sigma \theta\left(w_{0} P_{k, l} w_{0}^{-1}\right)=P_{n+2-l, n+2-k} .
$$

Moreover, if $\phi \in C_{c}^{\infty}\left(X_{n}\right)$, then

$$
\int_{U_{n+2-l, n+2-k}} \phi(u \cdot H) d u=\int_{U_{k, l}} \phi\left(\sigma \theta\left(w_{0} u\right) \cdot H\right) d u .
$$

Proof

From the identities

$$
w \cdot e_{i}=\left\{\begin{array}{ll}
e_{1} & i=1, \\
e_{n+1-i} & 2 \leq i \leq n-1, \\
e_{n} & i=n,
\end{array} \quad \text { and } \quad \sigma \theta e_{i}= \begin{cases}-e_{n} & i=1, \\
-e_{i} & 2 \leq i \leq n-1, \\
-e_{1} & i=n,\end{cases}\right.
$$

we obtain that $\sigma \theta\left(w \cdot \Sigma_{k, l}^{+}\right)=\Sigma_{n+2-l, n+2-k}^{+}$. This proves the first statement. The second statement follows from the first as

$$
U_{k, l} \rightarrow U_{n+2-l, n+2-k}, \quad u \mapsto \sigma \theta\left(w_{0} u w_{0}^{-1}\right)
$$

is a diffeomorphism with Jacobian 1.

\subsection{Reduction to an equivalent theorem}

The space $\mathcal{C}\left(X_{n}\right)$ is stable under translation by $G$ and pullback by $\sigma \theta$. Under the assumption that $P$ is of $\sigma$-parabolic rank 1, we conclude from Proposition 4.4, Lemma 4.6, and Lemma 4.7 that Theorem 3.1 is equivalent to the following theorem. 


\section{THEOREM 4.8}

Let $k$ and $l$ be integers such that $2 \leq k \leq l \leq n+2-k$. Then the integral

$$
\int_{U_{k, l}} \phi(u \cdot H) d u
$$

is absolutely convergent for every $\phi \in \mathcal{C}\left(X_{n}\right)$ if and only if $\frac{n+1}{2} \leq k \leq l \leq \frac{n+3}{2}$.

From now on we assume that $k$ and $l$ are integers such that $2 \leq k \leq l \leq n+2-k$.

\subsection{The integral}

\section{LEMMA 4.9}

Let $x, y \in \mathbb{R}^{n-2}$ and $z \in \mathbb{R}$. If $u_{x, y, z} \cdot H \in K a_{t} \cdot H$, then

$2 \cosh (4 t)$

$$
\begin{aligned}
= & (1-z+\langle x, y\rangle)^{2}(1+z)^{2}+(1-z+\langle x, y\rangle)^{2}\|y\|^{2}+(\langle x, y\rangle-z)^{2} \\
& +(1+z)^{2}\|x\|^{2}+2\langle x, y\rangle+\|x\|^{2}\|y\|^{2}+\|x\|^{2}+z^{2}+\|y\|^{2}+1
\end{aligned}
$$

We denote the orthogonal projection $\mathbb{R}^{n-2} \rightarrow\{0\}^{k-2} \times \mathbb{R}^{l-k} \times\{0\}^{n-l}$ by $\pi$. Let $x \in\{0\}^{k-2} \times \mathbb{R}^{n-k}, y \in \mathbb{R}^{l-2} \times\{0\}^{n-l}$, and $z \in \mathbb{R}$. If $u_{x, y, z} \cdot H \in K a_{t} \cdot H$, then

$$
2 \cosh (4 t)=\left\langle x, A_{y, z} x\right\rangle+\left\langle b_{y, z}, x\right\rangle+c_{y, z},
$$

where

$$
\begin{aligned}
A_{y, z} & =\left(\|y\|^{2}+(1+z)^{2}+1\right)\left(I_{n-2}+\pi(y) \pi(y)^{t}\right) \in \operatorname{Aut}\left(\mathbb{R}^{n-2}\right), \\
b_{y, z} & =2(1-z)\left(\|y\|^{2}+(1+z)^{2}+1\right) \pi(y) \in\{0\}^{k-2} \times \mathbb{R}^{l-k} \times\{0\}^{n-l}, \\
c_{y, z} & =(1-z)^{2}\left(\|y\|^{2}+(1+z)^{2}+1\right)+\left(z^{2}+2 z+\|y\|^{2}\right) \in \mathbb{R}_{\geq 2} .
\end{aligned}
$$

Proof

Straightforward computations show that

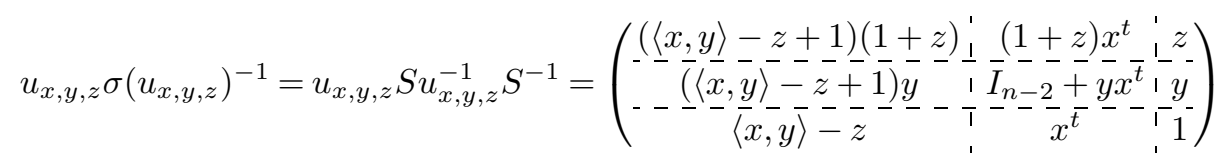

and

$$
\left\|I_{n-2}+y x^{t}\right\|_{H S}^{2}=n-2+2\langle x, y\rangle+\|x\|^{2}\|y\|^{2} .
$$

Now (4.13) follows from Lemma 2.1. Then (4.14) is a direct consequence of (4.13).

\section{COROLLARY 4.10}

Let $\phi \in C\left(X_{n}\right)$ be $K$-invariant and nonnegative. Let $\bar{\phi}: \mathbb{R}_{\geq 2} \rightarrow \mathbb{R}_{\geq 0}$ be given by

$$
\bar{\phi}(2 \cosh 4 t)=\phi\left(a_{t} \cdot H\right) .
$$


(Note that the function $\mathbb{R} \ni t \mapsto \phi\left(a_{t} \cdot H\right)$ is even since $\phi$ is $K$-invariant.) Furthermore, let $J_{k}: \mathbb{R}^{n-2} \times \mathbb{R} \rightarrow \mathbb{R}_{>0}$ be given by

$$
J_{k}(y, z)=\left(\|y\|^{2}+(1+z)^{2}+1\right)^{\frac{k-n}{2}}\left(1+\|\pi(y)\|^{2}\right)^{-\frac{1}{2}},
$$

and let $c^{\prime}: \mathbb{R}^{n-2} \times \mathbb{R} \rightarrow \mathbb{R}_{\geq 2}$ be given by

$$
c^{\prime}(y, z)=(1-z)^{2}\left(\|y\|^{2}+(1+z)^{2}+1\right) \frac{1}{1+\|\pi(y)\|^{2}}+\left(z^{2}+2 z+\|y\|^{2}\right) .
$$

Then

$$
\begin{aligned}
\int_{U_{k, l}} \phi(u \cdot H) d u= & \int_{z \in \mathbb{R}} \int_{y \in \mathbb{R}^{l-2} \times\{0\}^{n-l}} J_{k}(y, z) \\
& \times \int_{x \in\{0\}^{k-2} \times \mathbb{R}^{n-k}} \bar{\phi}\left(\|x\|^{2}+c^{\prime}(y, z)\right) d x d y d z .
\end{aligned}
$$

Proof

First, note that, by Lemma 4.6,

$$
\int_{U_{k, l}} \phi(u \cdot H) d u=\int_{z \in \mathbb{R}} \int_{y \in \mathbb{R}^{l-2} \times\{0\}^{n-l}} \int_{x \in\{0\}^{k-2} \times \mathbb{R}^{n-k}} \phi\left(u_{x, y, z} \cdot H\right) d x d y d z .
$$

We will use Lemma 4.9 to rewrite this integral. Note that the restriction $B_{y, z}$ of $A_{y, z}$ to $\{0\}^{k-2} \times \mathbb{R}^{n-k}$ is a positive definite symmetric automorphism of $\{0\}^{k-2} \times$ $\mathbb{R}^{n-k}$. We define $B_{y, z}^{1 / 2}$ and $B_{y, z}^{-1 / 2}$ to be the square root (defined in the usual way) and the inverse square root of $B_{y, z}$, respectively. Now we apply the substitution of variables $x^{\prime}=B_{y, z}^{1 / 2} x+\frac{1}{2} B_{y, z}^{-1 / 2} b_{y, z}$ to the inner integral. When $u_{x, y, z} \cdot H \in K a_{t} \cdot H$ we obtain from (4.14) that

$$
2 \cosh (4 t)=\left\|x^{\prime}\right\|^{2}+c_{y, z}^{\prime}
$$

where

$$
2 \leq c_{y, z}^{\prime}=c_{y, z}-\frac{1}{4}\left\|B_{y, z}^{-\frac{1}{2}} b_{y, z}\right\|^{2} .
$$

The Jacobian of the substitution equals the determinant of $B_{y, z}^{-1 / 2}$. In turn, this determinant equals $\left(\operatorname{det} B_{y, z}\right)^{-1 / 2}=J_{k}(y, z)$. Observing that $b_{y, z}$ is an eigenvector of $B_{y, z}$ with eigenvalue $\left(\|y\|^{2}+(1+z)^{2}+1\right)\left(1+\|\pi(y)\|^{2}\right)$, we see that

$$
\frac{1}{4}\left\|B_{y, z}^{-\frac{1}{2}} b_{y, z}\right\|^{2}=(1-z)^{2}\left(\|y\|^{2}+(1+z)^{2}+1\right) \frac{\|\pi(y)\|^{2}}{1+\|\pi(y)\|^{2}} .
$$

Hence, $c_{y, z}^{\prime}=c^{\prime}(y, z)$, and the corollary is proved.

\subsection{The case of convergence}

In view of Remark 2.3, the following proposition implies the if part of Theorem 4.8 .

PROPOSITION 4.11

Assume that $\frac{n+1}{2} \leq k \leq l \leq \frac{n+3}{2}$. For $m \in \mathbb{R}$, let $\phi_{m}: X_{n} \rightarrow \mathbb{R}$ be defined by 


$$
\phi_{m}\left(k^{\prime} a_{t} \cdot H\right):=(2 \cosh 4 t)^{\frac{1-n}{4}}(1+\log (2 \cosh (4 t)))^{-m} \quad\left(k^{\prime} \in K, t \in \mathbb{R}\right) .
$$

Then there exists $m \geq 0$ such that the integral $\int_{U_{k, l}} \phi_{m}(u \cdot H) d u$ is convergent.

\section{Proof}

Let $f_{m}(u)=u^{\frac{1-n}{4}}(1+\log u)^{-m}$ for $u \geq 1$. Then $f$ equals $\bar{\phi}_{m}$ as defined in Corollary 4.10 , and we see that it suffices to show that

$$
\begin{aligned}
I_{m} & :=\int_{z \in \mathbb{R}} \int_{y \in \mathbb{R}^{l-2} \times\{0\}^{n-l}} J_{k}(y, z) \int_{x \in\{0\}^{k-2} \times \mathbb{R}^{n-k}} f_{m}\left(\|x\|^{2}+c^{\prime}(y, z)\right) d x d y d z \\
& <\infty
\end{aligned}
$$

for $m \geq 0$ sufficiently large. We substitute $x=c^{\prime}(y, z)^{1 / 2} \xi$ in the inner integral and obtain that $I_{m}$ is equal to

$$
\begin{aligned}
& \int_{z \in \mathbb{R}} \int_{y \in \mathbb{R}^{l-2} \times\{0\}^{n-l}} J_{k}(y, z) c^{\prime}(y, z)^{\frac{n-k}{2}} \\
& \quad \times \int_{\xi \in\{0\}^{k-2} \times \mathbb{R}^{n-k}} f_{m}\left(c^{\prime}(y, z)\left(\|\xi\|^{2}+1\right)\right) d \xi d y d z .
\end{aligned}
$$

Observe that if $m=m_{1}+m_{2}$ with $m_{1}, m_{2} \geq 0$, then

$$
\left(1+\log \left[c^{\prime}(y, z)\left(\|\xi\|^{2}+1\right)\right]\right)^{-m} \leq\left(1+\log c^{\prime}(y, z)\right)^{-m_{2}}\left(1+\log \left(\|\xi\|^{2}+1\right)\right)^{-m_{1}} .
$$

Hence,

$$
\begin{aligned}
I_{m} \leq & \int_{z \in \mathbb{R}} \int_{y \in \mathbb{R}^{l-2} \times\{0\}^{n-l}} J_{k}(y, z) c^{\prime}(y, z)^{\frac{n+1}{4}-\frac{k}{2}}\left(1+\log c^{\prime}(y, z)\right)^{-m_{2}} d y d z \\
& \times \int_{\xi \in\{0\}^{k-2} \times \mathbb{R}^{n-k}}\left(\|\xi\|^{2}+1\right)^{\frac{1-n}{4}}\left(1+\log \left(\|\xi\|^{2}+1\right)\right)^{-m_{1}} d \xi
\end{aligned}
$$

Since $k \geq \frac{n+1}{2}$, we have $\frac{n-1}{2} \geq n-k$ and see that the integral over $\xi$ converges for $m_{1} \geq 2$ (using polar coordinates).

For the integral over $(y, z)$ we shall need the following estimate of $c^{\prime}(y, z)$. We write $y=(v, w, 0)$, where $v \in \mathbb{R}^{k-2}$ and $w=\pi(y) \in \mathbb{R}^{l-k}$, and claim that

$$
c^{\prime}(y, z) \geq \frac{1}{4}\left(\|w\|^{2}+1\right)^{-1}\left((1-z)^{2}+1\right)\left(\|v\|^{2}+(1+z)^{2}+1\right)+\|w\|^{2}+1
$$

To verify the claim, we note that

$$
\begin{aligned}
c^{\prime}(y, z)= & \left(\|w\|^{2}+1\right)^{-1}(1-z)^{2}\left(\|v\|^{2}+\|w\|^{2}+(1+z)^{2}+1\right) \\
& +z^{2}+2 z+\|v\|^{2}+\|w\|^{2} \\
= & \left(\|w\|^{2}+1\right)^{-1}\left(\left((1-z)^{2}+1+\|w\|^{2}\right)\|v\|^{2}+z^{4}+1+2 z^{2}\|w\|^{2}\right) \\
& +\|w\|^{2}+1 \\
\geq & \left(\|w\|^{2}+1\right)^{-1}\left(\left((1-z)^{2}+1\right)\|v\|^{2}+z^{4}+1\right)+\|w\|^{2}+1 \\
\geq & \frac{1}{4}\left(\|w\|^{2}+1\right)^{-1}\left(\left((1-z)^{2}+1\right)\|v\|^{2}+z^{4}+4\right)+\|w\|^{2}+1 .
\end{aligned}
$$


Using

$$
\left((1-z)^{2}+1\right)\left((1+z)^{2}+1\right)=z^{4}+4,
$$

we obtain the validity of claim (4.16).

We first assume that $k=l$. Then $w=0$ and we obtain

$$
c^{\prime}(y, z) \geq \frac{1}{4}\left((1-z)^{2}+1\right)\left(\|v\|^{2}+(1+z)^{2}+1\right)+1
$$

and

$$
J_{k}(y, z)=\left(\|v\|^{2}+(1+z)^{2}+1\right)^{\frac{k-n}{2}} .
$$

Hence, there exists a constant $C>0$, independent of $m$, such that

$$
\begin{aligned}
I_{m} \leq & C \int_{z \in \mathbb{R}} \int_{v \in \mathbb{R}^{k-2}}\left(\|v\|^{2}+(1+z)^{2}+1\right)^{\frac{1-n}{4}}\left((1-z)^{2}+1\right)^{\frac{n+1}{4}-\frac{k}{2}} \\
& \times\left(1+\log \left[1+\frac{1}{4}\left((1-z)^{2}+1\right)\left(\|v\|^{2}+(1+z)^{2}+1\right)\right]\right)^{-m_{2}} d v d z .
\end{aligned}
$$

The substitution of $v=\left((1+z)^{2}+1\right)^{1 / 2} \eta$ now allows us to estimate $I_{m}$ by a constant times the product of the integrals

$$
\int_{\eta \in \mathbb{R}^{k-2}}\left(\|\eta\|^{2}+1\right)^{\frac{1-n}{4}}\left(1+\log \left(\|\eta\|^{2}+1\right)\right)^{-m_{3}} d y
$$

and

$$
\int_{z \in \mathbb{R}}\left((1+z)^{2}+1\right)^{\frac{k}{2}-\frac{n+3}{4}}\left((1-z)^{2}+1\right)^{\frac{n+1}{4}-\frac{k}{2}}\left(1+\log \left(\frac{1}{4} z^{4}+2\right)\right)^{-m_{4}} d z
$$

where $m_{2}=m_{3}+m_{4}$. In the above we already saw that (4.17) converges for $m_{3} \geq 2$. The remaining integral is easily seen to converge for $m_{4} \geq 2$.

Next we assume $k<l$. Then $n$ is odd, $k=\frac{n+1}{2}$, and $l-k=1$. Hence, the power of $c^{\prime}(y, z)$ in the first integral in (4.15) equals zero. We thus see that $I_{m}$ is bounded by a constant times

$$
\begin{aligned}
\int_{z \in \mathbb{R}} & \int_{v \in \mathbb{R}^{k-2}} \int_{w \in \mathbb{R}}\left(\|v\|^{2}+w^{2}+(1+z)^{2}+1\right)^{\frac{1-n}{4}} \\
& \times\left(w^{2}+1\right)^{-\frac{1}{2}}\left(1+\log c^{\prime}(y, z)\right)^{-m_{2}} d w d v d z
\end{aligned}
$$

Furthermore,

$$
c^{\prime}(y, z) \geq \frac{1}{4}\left(w^{2}+1\right)^{-1}\left(\|v\|^{2}+(1+z)^{2}+1\right)+w^{2}+1 .
$$

The substitutions $v=\left(w^{2}+1\right)^{1 / 2} \eta$ and $1+z=\left(w^{2}+1\right)^{1 / 2} \zeta$ then allow us to estimate by the product of

$$
\int_{(\eta, \zeta) \in \mathbb{R}^{k-2} \times \mathbb{R}}\left(\|\eta\|^{2}+\zeta^{2}+1\right)^{\frac{1-n}{4}}\left(1+\log \left(\|\eta\|^{2}+\zeta^{2}+1\right)\right)^{-m_{3}} d(\eta, \zeta)
$$

and

$$
\int_{w \in \mathbb{R}}\left(w^{2}+1\right)^{-\frac{1}{2}}\left(1+\log \left(w^{2}+1\right)\right)^{-m_{4}} d w
$$


where $m_{2}=m_{3}+m_{4}$. Both integrals are easily seen to converge for $m_{3}, m_{4} \geq 2$.

\subsection{The case of divergence}

The following proposition and its corollary imply the only if part of Theorem 4.8.

PROPOSITION 4.12

Let $\nu \in \mathbb{R}$, and let $\phi_{\nu}$ be the function $X_{n} \rightarrow \mathbb{R}$ given by

$$
\phi_{\nu}\left(k^{\prime} a_{t} \cdot H\right)=(2 \cosh 4 t)^{\nu} \quad\left(k^{\prime} \in K, t \in \mathbb{R}\right) .
$$

Then the integral $\int_{U_{k, l}} \phi_{\nu}(u \cdot H) d u$ is divergent for $\nu>\min \left\{\frac{k-n}{2}, \frac{2-l}{2}\right\}$.

Proof

Let $\bar{\phi}_{\nu}$ be the function associated to $\phi_{\nu}$ as in Corollary 4.10. Then $\bar{\phi}_{\nu}(r)=r^{\nu}$ for $r \geq 1$. Clearly, the integral

$$
\int_{x \in \mathbb{R}^{n-k}}\left(\|x\|^{2}+c\right)^{\nu} d x
$$

is divergent for all positive constants $c$ if $\nu>\frac{k-n}{2}$. Hence, it follows from Corollary 4.10 that $\int_{U_{k, l}} \phi_{\nu}(u \cdot H) d u$ is divergent for $\operatorname{such} \nu$. Note that $\phi_{\nu}$ satisfies

$$
\phi_{\nu}(\sigma \theta(k g) \cdot H)=\phi_{\nu}(g \cdot H) \quad(g \in G, k \in K) .
$$

Combining this with Lemma 4.7, we infer that

$$
\int_{U_{n+2-l, n+2-k}} \phi_{\nu}(u \cdot H) d u=\int_{U_{k, l}} \phi_{\nu}(u \cdot H) d u .
$$

By the previous argument, we obtain that the first (hence, also the second) integral is divergent for $\nu>\frac{(n+2-l)-n}{2}=\frac{2-l}{2}$. The assertion now follows.

COROLLARY 4.13

Assume that $k<\frac{n+1}{2}$ or $l>\frac{n+3}{2}$. Then there exists a function $\phi \in \mathcal{C}\left(X_{n}\right)$ such that $\int_{U_{k, l}} \phi(u \cdot H) d u$ is divergent.

Proof

Assume that $k<\frac{n+1}{2}$ or $l>\frac{n+3}{2}$. Then $\min \left\{\frac{k-n}{2}, \frac{2-l}{2}\right\}<\frac{1-n}{4}$. We may therefore take $\nu$ such that $\min \left\{\frac{k-n}{2}, \frac{2-l}{2}\right\}<\nu<\frac{1-n}{4}$. Then $\phi_{\nu} \in \mathcal{C}\left(X_{n}\right)$ by Lemma 2.4. The result now follows by application of Proposition 4.12.

\section{Proof of Theorem 3.1 for $\sigma$-parabolic rank 0}

We now turn to the proof of Theorem 3.1 under the assumption that $P$ is of $\sigma$-parabolic rank 0. In Sections 5.1-5.4 we first reduce the statement to a (seemingly) less general statement, which we then prove in Sections 5.5-5.7. 


\subsection{Root systems}

Recall the element $\kappa$ from $(2.1)$. We define $\mathfrak{b}=\operatorname{Ad}(\kappa) \mathfrak{a}$. Note that $\mathfrak{b}$ is a Cartan subalgebra of $\mathfrak{g}$ and that $\mathfrak{a}_{\mathfrak{h}} \subseteq \mathfrak{b} \subseteq \mathfrak{h}$. Furthermore, $\mathfrak{b}$ is a Cartan subalgebra of $\mathfrak{h}$ as well. We write $B$ for $\exp (\mathfrak{b})$.

Recall the functionals $e_{k}$ from Section 4.1. For $1 \leq k \leq n$ we define $f_{k}: \mathfrak{b} \rightarrow \mathbb{R}$ by $f_{k}=e_{k} \circ \operatorname{Ad}(\kappa)^{-1}$. The root system of $\mathfrak{b}$ in $\mathfrak{g}$ then equals

$$
\bar{\Sigma}:=\Sigma(\mathfrak{g}, \mathfrak{b})=\left\{f_{i}-f_{j}: 1 \leq i, j \leq n, i \neq j\right\} .
$$

The associated root spaces are given by $\mathfrak{g}_{f_{i}-f_{j}}=\mathbb{R}\left(\operatorname{Ad}(\kappa) E_{i, j}\right)$.

Note that

$$
\bar{\Sigma}_{\mathfrak{h}}:=\Sigma_{\mathfrak{h}}(\mathfrak{b})=\left\{f_{i}-f_{j}: 1 \leq i, j \leq n-1, i \neq j\right\}
$$

is both the root system of $\mathfrak{b}$ in $\mathfrak{h}$ and the set of $\mathfrak{h}$-roots in $\bar{\Sigma}$. Let

$$
\bar{\Sigma}_{\mathfrak{h}}^{+}=\left\{f_{i}-f_{j}: 1 \leq i<j \leq n-1\right\} .
$$

Then $\bar{\Sigma}_{\mathfrak{h}}^{+}$is a positive system for $\bar{\Sigma}_{\mathfrak{h}}$. Finally, we define

$$
\bar{\rho}_{\mathfrak{h}}:=\frac{1}{2} \sum_{\alpha \in \bar{\Sigma}_{\mathfrak{h}}^{+}} \alpha .
$$

\subsection{Classification of parabolic subgroups}

Recall that $\mathcal{Q}$ denotes the set of minimal parabolic subgroups containing a maximal connected split abelian subgroup that is contained in $H$. Furthermore, $\mathcal{Q}(\mathfrak{b})$ denotes the subset of $\mathcal{Q}$ consisting of minimal parabolic subgroups containing $B$. Given $Q \in \mathcal{P}(\mathfrak{b})$, we agree to use the abbreviation $\bar{\Sigma}(Q):=\Sigma(\mathfrak{b} ; Q)$.

\section{LEMMA 5.1}

Let $P \in \mathcal{Q}$. Then there exists a parabolic subgroup $Q \in \mathcal{Q}(\mathfrak{b})$ which is $H$-conjugate to $P$ and satisfies

$$
\bar{\Sigma}_{\mathfrak{h}}^{+} \subseteq \bar{\Sigma}(Q) .
$$

Let $Q$ be any such parabolic subgroup. Then $\rho_{Q, \mathfrak{h}}=\bar{\rho}_{\mathfrak{h}}$. Moreover, $P$ is $\mathfrak{h}$ compatible if and only if $Q$ is $\mathfrak{h}$-compatible (see Definition 2.5).

\section{Proof}

Since $P \in \mathcal{Q}$, it follows from Corollaries 1.10 and 1.13 that $P$ is $H$-conjugate to a minimal parabolic subgroup $Q \in \mathcal{Q}(\mathfrak{b})$ satisfying (5.2). The latter condition implies that $\rho_{Q, \mathfrak{h}}=\bar{\rho}_{\mathfrak{h}}$. The final statement follows from Remark 2.6.

We will now classify the parabolic subgroups $Q \in \mathcal{Q}(\mathfrak{b})$ satisfying (5.2). The assignment $Q \mapsto \bar{\Sigma}(Q)$ defines a bijection from the set $\mathcal{P}(\mathfrak{b})$ onto the set of positive systems for $\bar{\Sigma}$. In turn, the latter set is in bijective correspondence with the permutation group $S_{n}$. For a given $\tau \in S_{n}$, the associated positive system is given by

$$
\bar{\Sigma}(Q)=\left\{f_{\tau^{-1}(i)}-f_{\tau^{-1}(j)}: 1 \leq i<j \leq n\right\} .
$$


Equivalently, a root $f_{i}-f_{j} \in \bar{\Sigma}$ belongs to $\bar{\Sigma}(Q)$ if and only if $\tau(i)<\tau(j)$. We infer that the parabolic subgroups $Q \in \mathcal{Q}(\mathfrak{b})$ satisfying (5.2) correspond to the permutations $\tau \in S_{n}$ satisfying

$$
\tau(i)<\tau(j) \text { for } 1 \leq i<j \leq n-1 .
$$

Given such a permutation $\tau \in S_{n}$, there exists a unique $k$, with $1 \leq k \leq n$, such that $\tau(n)=k$. Conversely, for each integer $k$ with $1 \leq k \leq n$, there exists a unique $\tau \in S_{n}$ satisfying (5.3) and $\tau(n)=k$. From now on we write $Q_{k}$ for the corresponding minimal parabolic subgroup and $\bar{\Sigma}_{k}^{+}$for $\bar{\Sigma}\left(Q_{k}\right)$. Moreover, we write $N_{k}$ for $N_{Q_{k}}$. For future reference we note that the positive system determined by $k$ is given by the disjoint union

$$
\bar{\Sigma}_{k}^{+}=\bar{\Sigma}_{\mathfrak{h}}^{+} \cup\left\{f_{i}-f_{n}: 1 \leq i \leq k-1\right\} \cup\left\{f_{n}-f_{i}: k \leq i \leq n-1\right\} .
$$

We now provide $\mathfrak{b}^{*}$ with the inner product that turns $\operatorname{Ad}(\kappa)^{*}: \mathfrak{b}^{*} \rightarrow \mathfrak{a}^{*}$ into an isometry (see (4.7) for the description of the inner product on $\mathfrak{a}^{*}$ ).

\section{LEMMA 5.2}

Let $i$ and $j$ be integers such that $1 \leq i<j \leq n$. Then

$$
\left\langle f_{i}-f_{j}, \bar{\rho}_{\mathfrak{h}}\right\rangle= \begin{cases}j-i & \text { if } 1 \leq i<j \leq n-1, \\ \frac{n}{2}-i & \text { if } 1 \leq i \leq n-1 \text { and } j=n .\end{cases}
$$

In particular, $\left\langle\alpha, \bar{\rho}_{\mathfrak{h}}\right\rangle>0$ for every $\alpha \in \bar{\Sigma}_{k}^{+}$if and only if $\frac{n}{2}<k<\frac{n}{2}+1$, that is, if and only if $n$ is odd and $k=\frac{n+1}{2}$.

Proof

Using the definition of $\bar{\rho}_{\mathfrak{h}}$, we find

$$
2 \bar{\rho}_{\mathfrak{h}}=\sum_{i=1}^{n-1}(n-2 i) f_{i} .
$$

The first statement follows directly from this formula, and the second follows from comparison with (5.4).

Combining the previous lemmas, we now arrive at the following proposition.

\section{PROPOSITION 5.3}

Let $Q \in \mathcal{Q}$. Then there exists a unique integer $k$, with $1 \leq k \leq n$, such that $Q$ is $H$-conjugate to $Q_{k}$. Moreover, $Q$ is $\mathfrak{h}$-compatible if and only if $n$ is odd and $k=\frac{n+1}{2}$.

Proof

Only the uniqueness remains to be proved. Let $1 \leq k, k^{\prime} \leq n$, and assume that there exists an $h \in H$ such that $h Q_{k} h^{-1}=Q_{k^{\prime}}$. Then $B$ and $h B h^{-1}$ are both $\sigma$-stable split components of $Q_{k^{\prime}}$. From Theorem 1.8 it follows that there exists a unique $n \in N_{k^{\prime}} \cap H$ such that $n h B h^{-1} n^{-1}=B$. Let $h^{\prime}=n h$. Then $h^{\prime} \in N_{H}(\mathfrak{b})$ 
and $h^{\prime} Q_{k} h^{\prime-1}=Q_{k^{\prime}}$. Now $h^{\prime}$ induces an element $w$ in the Weyl group of the root system $\bar{\Sigma}_{\mathfrak{h}}$. Since $\bar{\Sigma}_{\mathfrak{h}}^{+}$is a positive system for $\bar{\Sigma}_{\mathfrak{h}}$ and $\bar{\Sigma}_{\mathfrak{h}}^{+}$is contained in both $\bar{\Sigma}_{k}^{+}$and $\bar{\Sigma}_{k^{\prime}}^{+}$, it follows that $w$ acts trivially on $\bar{\Sigma}_{\mathfrak{h}}^{+}$and hence that $h^{\prime}$ acts trivially on $\mathfrak{b}$. We conclude that $h Q_{k} h^{-1}=Q_{k^{\prime}}$ and therefore $k=k^{\prime}$. This proves uniqueness.

\subsection{Decomposition and invariant measures}

Let $k$ be an integer such that $1 \leq k \leq n$.

\section{LEMMA 5.4}

Let $\mathfrak{n}_{k}$ be the Lie algebra of $N_{k}$. Then

$$
\begin{aligned}
& \mathfrak{n}_{k} \cap \mathfrak{h}=\bigoplus_{\alpha \in \bar{\Sigma}_{\mathfrak{h}}^{+}} \mathfrak{g}_{\alpha}=\bigoplus_{1 \leq i<j \leq n-1} \mathbb{R}\left(\operatorname{Ad}(\kappa) E_{i, j}\right) \\
& \mathfrak{n}_{k} \cap \mathfrak{q}=\bigoplus_{\alpha \in \bar{\Sigma} \backslash \bar{\Sigma}_{\mathfrak{h}}^{+}} \mathfrak{g}_{\alpha}=\bigoplus_{1 \leq i \leq k-1} \mathbb{R}\left(\operatorname{Ad}(\kappa) E_{i, n}\right) \oplus \bigoplus_{k \leq i \leq n-1} \mathbb{R}\left(\operatorname{Ad}(\kappa) E_{n, i}\right) .
\end{aligned}
$$

Proof

Let $\alpha \in \bar{\Sigma}$. Since $\sigma \alpha=\alpha$ and $\mathfrak{g}_{\alpha}$ is 1-dimensional, we have either $\mathfrak{g}_{\alpha} \subseteq \mathfrak{h}$ or $\mathfrak{g}_{\alpha} \subseteq \mathfrak{q}$. By definition, the first is the case for $\alpha \in \bar{\Sigma}_{\mathfrak{h}}$ and the latter for $\alpha \in \bar{\Sigma} \backslash \bar{\Sigma}_{\mathfrak{h}}$. The lemma now follows from (5.4).

We write $V_{k}$ for the submanifold $\exp \left(\mathfrak{n}_{k} \cap \mathfrak{q}\right)$ of $N_{k}$. For $x, y \in \mathbb{R}^{n-1}$ with $\langle x, y\rangle=0$ we define

$$
v_{x, y}=\kappa \exp \left(\begin{array}{c:c}
0 & x \\
\hdashline y & 0
\end{array}\right) \kappa^{-1}=\kappa\left(\begin{array}{c:c}
I_{n-1}+\frac{1}{2} x y^{t} & x \\
\hdashline y^{t} & 1
\end{array}\right) \kappa^{-1} .
$$

A straightforward computation shows that

$$
V_{k}=\left\{v_{x, y}: x \in \mathbb{R}^{k-1} \times\{0\}^{n-k}, y \in\{0\}^{k-1} \times \mathbb{R}^{n-k}\right\} .
$$

We equip $V_{k}$ with the pushforward along exp of the Lebesgue measure on $\mathfrak{n}_{k} \cap \mathfrak{q}$. Then the following lemma is a direct consequence of [5, Proposition 1.1] and Lemma 5.4 .

\section{LEMMA 5.5}

The map

$$
V_{k} \times\left(N_{k} \cap H\right) \rightarrow N_{k}, \quad(v, n) \mapsto v n
$$

is a diffeomorphism. Moreover, there exists a normalization for the invariant measure $d x$ on $N_{k} /\left(N_{k} \cap H\right)$ such that, for every $\phi \in C_{c}^{\infty}\left(N_{k} /\left(N_{k} \cap H\right)\right)$,

$$
\int_{N_{k} /\left(N_{k} \cap H\right)} \phi(x) d x=\int_{V_{k}} \phi\left(v \cdot\left(N_{k} \cap H\right)\right) d v .
$$




\subsection{Reduction to an equivalent theorem}

Under the assumption that $P$ is of $\sigma$-parabolic rank 0 , it follows from Proposition 5.3 and Lemma 5.5 that Theorem 3.1 is equivalent to the following theorem.

\section{THEOREM 5.6}

Let $k$ be an integer with $1 \leq k \leq n$. Then the integral

$$
\int_{V_{k}} \phi(v \cdot H) d v
$$

is absolutely convergent for every $\phi \in \mathcal{C}\left(X_{n}\right)$ if and only if $k=\frac{n+1}{2}$. In particular, if $n$ is even, then for every $k$ there exists $\phi \in \mathcal{C}\left(X_{n}\right)$ such that the integral is divergent.

\subsection{The integral}

Recall (5.5) for the definition of $v_{x, y}$.

LEMMA 5.7

Let $x, y \in \mathbb{R}^{n-1}$ with $\langle x, y\rangle=0$. If $v_{x, y} \cdot H \in K a_{t} \cdot H$, then

$$
2 \cosh (4 t)=2+4\|x\|^{2}+4\|y\|^{2}+4\|x\|^{2}\|y\|^{2} .
$$

Proof

By straightforward computations we see that

$$
v_{x, y} \sigma\left(v_{x, y}\right)^{-1}=v_{x, y}^{2}=v_{2 x, 2 y}=\kappa\left(\begin{array}{c:c}
I_{n-1}+2 x y^{t} & 2 x \\
\hdashline-y^{t^{-}} & 1
\end{array}\right) \kappa^{-1}
$$

and

$$
\left\|I_{n-1}+2 y x^{t}\right\|_{H S}^{2}=n-1+4\|x\|^{2}\|y\|^{2} .
$$

Therefore,

$$
\left\|v_{x, y} \sigma\left(v_{x, y}\right)^{-1}\right\|_{H S}^{2}=n+4\|x\|^{2}+4\|y\|^{2}+4\|x\|^{2}\|y\|^{2} .
$$

The lemma now follows from Lemma 2.1.

\section{COROLLARY 5.8}

Let $\phi \in C\left(X_{n}\right)$ be $K$-invariant and nonnegative. As in Corollary 4.10, let $\bar{\phi}$ : $\mathbb{R}_{\geq 2} \rightarrow \mathbb{R}_{\geq 0}$ be defined by

$$
\bar{\phi}(2 \cosh 4 t)=\phi\left(a_{t} \cdot H\right) .
$$

Then

$$
\int_{V_{k}} \phi(v \cdot H) d v=\int_{\mathbb{R}^{k-1}} \int_{\mathbb{R}^{n-k}} \bar{\phi}\left(2+4\|x\|^{2}+4\|y\|^{2}+4\|x\|^{2}\|y\|^{2}\right) d y d x .
$$

Proof

Let $x \in \mathbb{R}^{k-1} \times\{0\}^{n-k}$, let $y \in\{0\}^{k-1} \times \mathbb{R}^{n-k}$, and assume that $v_{x, y} \in k^{\prime} a_{t} H$, for $k^{\prime} \in K$ and $t \in \mathbb{R}$. Then it follows that 


$$
\phi\left(v_{x, y} \cdot H\right)=\bar{\phi}(2 \cosh 4 t)=\bar{\phi}\left(2+4\|x\|^{2}+4\|y\|^{2}+4\|x\|^{2}\|y\|^{2}\right),
$$

by Lemma 5.7 .

\subsection{The case of convergence}

In view of Remark 2.3, the following proposition implies the if part of Theorem 5.6.

\section{PROPOSITION 5.9}

Assume that $k=\frac{n+1}{2}$. For $m \in \mathbb{R}$, let $\phi_{m}: X_{n} \rightarrow \mathbb{R}$ be given by

$$
\phi_{m}\left(k^{\prime} a_{t} \cdot H\right)=(2 \cosh 4 t)^{\frac{1-n}{4}}(1+\log (2 \cosh 4 t))^{-m} \quad\left(k^{\prime} \in K, t \in \mathbb{R}\right) .
$$

Then there exists $m \geq 0$ such that the integral $\int_{V_{k}} \phi_{m}(v \cdot H) d v$ is absolutely convergent.

\section{Proof}

Let $\bar{\phi}_{m}$ be defined in terms of $\bar{\phi}$ as in Corollary 5.8. Then, clearly, $\bar{\phi}_{m}(z)=$ $z^{\frac{1-n}{4}}(1+\log z)^{-m}$ from which we see that $\bar{\phi}_{m}$ is a decreasing function of $z \geq 1$. Hence,

$$
\left.\bar{\phi}_{m}\left(2+4\|x\|^{2}+4\|y\|^{2}+4\|x\|^{2}\|y\|^{2}\right)\right) \leq \bar{\phi}_{m}\left(1+\|x\|^{2}+\|y\|^{2}+\|x\|^{2}\|y\|^{2}\right),
$$

for $x \in \mathbb{R}^{k-1}=\mathbb{R}^{\frac{n-1}{2}}, y \in \mathbb{R}^{n-k}=\mathbb{R}^{\frac{n-1}{2}}$, and by the mentioned corollary we see that

$$
\begin{aligned}
\int_{V_{\frac{n+1}{2}}} & \phi_{m}(v \cdot H) d v \\
\leq & \int_{\mathbb{R}^{\frac{n-1}{2}}} \int_{\mathbb{R}^{\frac{n-1}{2}}} \bar{\phi}_{m}\left(1+\|x\|^{2}+\|y\|^{2}+\|x\|^{2}\|y\|^{2}\right) d y d x \\
= & \int_{\mathbb{R}^{\frac{n-1}{2}}} \int_{\mathbb{R}^{\frac{n-1}{2}}} \frac{\left(1+\|x\|^{2}\right)^{\frac{1-n}{4}}\left(1+\|y\|^{2}\right)^{\frac{1-n}{4}}}{\left(1+\log \left(1+\|x\|^{2}+\|y\|^{2}+\|x\|^{2}\|y\|^{2}\right)\right)^{m}} d y d x .
\end{aligned}
$$

Since

$$
\left(1+\log \left(1+\|x\|^{2}+\|y\|^{2}+\|x\|^{2}\|y\|^{2}\right)\right)^{2} \geq\left(1+\log \left(1+\|x\|^{2}\right)\right)\left(1+\log \left(1+\|y\|^{2}\right)\right),
$$

the last double integral is at most

$$
\left(\int_{\mathbb{R}^{\frac{n-1}{2}}} \frac{\left(1+\|x\|^{2}\right)^{\frac{1-n}{4}}}{\left(1+\log \left(1+\|x\|^{2}\right)\right)^{\frac{m}{2}}} d r\right)^{2} .
$$

By using polar coordinates, one readily verifies that the integral in this expression is absolutely convergent for $m>2$.

\subsection{The case of divergence}

The following proposition and its corollary imply the only if part of Theorem 5.6. 
PROPOSITION 5.10

Let $\nu \in \mathbb{R}$, and let $\phi_{\nu}: X_{n} \rightarrow \mathbb{R}$ be given by

$$
\phi_{\nu}\left(k^{\prime} a_{t} \cdot H\right)=(2 \cosh 4 t)^{\nu} \quad\left(k^{\prime} \in K, t \in \mathbb{R}\right) .
$$

Then the integral $\int_{V_{k}} \phi_{\nu}(v \cdot H) d v$ is divergent for $\nu \geq \min \left\{\frac{1-k}{2}, \frac{k-n}{2}\right\}$.

Proof

The function $\bar{\phi}_{\nu}$ associated to $\phi_{\nu}$ as in Corollary 5.8 is given by $z \mapsto z^{\nu}$. By the mentioned corollary we obtain

$$
\int_{V_{k}} \phi_{\nu}(v \cdot H) d v=\int_{\mathbb{R}^{k-1}} \int_{\mathbb{R}^{n-k}}\left(2+4\|x\|^{2}+4\|y\|^{2}+4\|x\|^{2}\|y\|^{2}\right)^{\nu} d y d x .
$$

Clearly the integral on the right-hand side is divergent if $\nu \geq 0$. We assume that $\nu<0$. Then the integral on the right-hand side is larger than

$$
\begin{gathered}
4^{\nu} \int_{\mathbb{R}^{k-1}} \int_{\mathbb{R}^{n-k}}\left(1+\|x\|^{2}+\|y\|^{2}+\|x\|^{2}\|y\|^{2}\right)^{\nu} d y d x \\
=4^{\nu} \int_{\mathbb{R}^{k-1}}\left(1+\|x\|^{2}\right)^{\nu} d x \int_{\mathbb{R}^{n-k}}\left(1+\|y\|^{2}\right)^{\nu} d y .
\end{gathered}
$$

The condition on $\nu$ implies that $2 \nu \geq 1-k$ or $2 \nu \geq k-n$. By using polar coordinates, we see that one of the integrals in (5.7) diverges. This completes the proof.

\section{COROLLARY 5.11}

Assume that $k \neq \frac{n+1}{2}$. Then there exists a function $\phi \in \mathcal{C}\left(X_{n}\right)$ such that $\int_{V_{k}} \phi(v \cdot H) d v$ is divergent.

Proof

Assume that $k \neq \frac{n+1}{2}$. Then $\min \left\{\frac{1-k}{2}, \frac{k-n}{2}\right\}<\frac{1-n}{4}$, and we may select $\nu$ such that

$$
\min \left\{\frac{1-k}{2}, \frac{k-n}{2}\right\}<\nu<\frac{1-n}{4} .
$$

Then $\phi_{\nu} \in \mathcal{C}\left(X_{n}\right)$ by Lemma 2.4. The claim now follows from Proposition 5.10.

\section{Proof of Theorem 3.2}

\subsection{Reduction to an equivalent theorem}

By Propositions 4.4 and 5.3 it suffices to prove the claims in Theorem 3.2 for $P=P_{k, l}$ with $\frac{n+1}{2} \leq k=l \leq \frac{n+3}{2}$ and (for $n$ odd) $Q=Q_{\frac{n+1}{2}}$ only.

We recall the definition of $a_{t}$ from (2.2). An easy computation shows that

$$
\delta_{P_{k, k}}\left(a_{t}\right)=e^{t} \text {. }
$$

It follows from Theorem 4.8 that for all $\phi \in \mathcal{C}\left(X_{n}\right)$ the integral

$$
\int_{U_{k, k}} \phi\left(a_{s} u \cdot H\right) d u
$$


yields a well-defined function of $s \in \mathbb{R}$. We are interested in the decay of this function or, more precisely, of the modified function

$$
s \mapsto e^{s} \int_{U_{k, k}} \phi\left(a_{s} u \cdot H\right) d u
$$

and will prove the following result, which implies Theorem 3.2.

\section{THEOREM 6.1}

Assume that $\frac{n+1}{2} \leq k \leq \frac{n+3}{2}$. Let $\phi \in \mathcal{C}\left(X_{n}\right)$.

(i) If $n$ is even, then for every $N \in \mathbb{N}$ there exist $c>0$ and $m \in \mathbb{N}$ such that, for every $s \in \mathbb{R}$,

$$
\left|e^{s} \int_{U_{k, k}} \phi\left(a_{s} u \cdot H\right) d u\right| \leq c(1+|s|)^{-N} \mu_{1, m}(\phi) .
$$

(ii) If $n$ is odd, then for every $R \in \mathbb{R}$ and $N \in \mathbb{N}$ there exist a $c>0$ and $m \in \mathbb{N}$ such that, for every $s \in \mathbb{R}$ with $s<R$,

$$
\left|e^{s} \int_{U_{k, k}} \phi\left(a_{s} u \cdot H\right) d u\right| \leq c(1+|s|)^{-N} \mu_{1, m}(\phi) .
$$

Furthermore, for $s$ moving in the other direction, there exists an element $\kappa_{0} \in K$, independent of $\phi$, such that

$$
\lim _{s \rightarrow \infty} e^{s} \int_{U_{k, k}} \phi\left(a_{s} u \cdot H\right) d u=\int_{V_{\frac{n+1}{2}}} \phi\left(\kappa_{0} v \cdot H\right) d v .
$$

In particular, the limit exists and is nonzero as a function of $\phi$.

\subsection{Proof of Theorem 6.1}

We recall the definition of $u_{x, y, z}$ from (4.9) and start with a few lemmas.

\section{LEMMA 6.2}

Let $s \in \mathbb{R}, x \in \mathbb{R}^{n-k}, y \in \mathbb{R}^{k-2}$, and $z \in \mathbb{R}$. If $a_{s} u_{x, y, z} \cdot H \in K a_{t} \cdot H$, then

$$
2 \cosh (4 t)=f_{1}+f_{2}\|x\|^{2}+f_{3}\|y\|^{2}+\|x\|^{2}\|y\|^{2},
$$

where

$$
\begin{aligned}
& f_{1}=f_{1}(s, z)=e^{4 s}(1-z)^{2}(1+z)^{2}+e^{-4 s}+2 z^{2}, \\
& f_{2}=f_{2}(s, z)=e^{2 s}(1+z)^{2}+e^{-2 s}, \\
& f_{3}=f_{3}(s, z)=e^{2 s}(1-z)^{2}+e^{-2 s} .
\end{aligned}
$$

Proof

From (4.9) we find

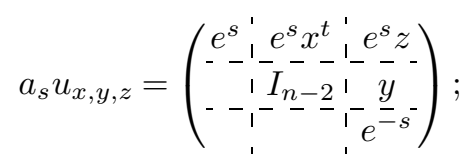


hence,

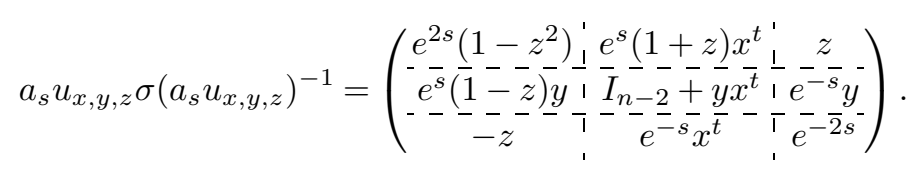

The proof is completed by combining Lemma 2.1 with a straightforward computation of the squared Hilbert-Schmidt norm of the last matrix, analogous to the computation in the proof of Lemma 4.9. (Note that now $\langle x, y\rangle=0$ since $k=l$.)

We shall need some estimates for $f_{1}, f_{2}$, and $f_{3}$.

\section{LEMMA 6.3}

Let $s, z \in \mathbb{R}$, and let $f_{i}=f_{i}(s, z)$ be as above for $i=1,2,3$. Then

$$
f_{2} f_{3}=f_{1}+2, \quad 2 \leq f_{1}, \quad f_{1} \leq f_{2} f_{3} \leq 2 f_{1},
$$

and

$$
1+z^{2} \leq f_{1}
$$

\section{Proof}

The equality in (6.8) is easily verified, and the lower bound for $f_{1}$ follows from (6.4) with $x=y=0$. Then, $f_{1} \leq f_{1}+2 \leq 2 f_{1}$ implies the final statement in (6.8). Finally we observe that in addition to $2 \leq f_{1}$ we also have $2 z^{2} \leq f_{1}$, whence (6.9).

\section{LEMMA 6.4}

Let $R \in \mathbb{R}$. Then there exists a constant $A>0$ such that

$$
f_{i}(s, z) \geq A\left(e^{2 s} z^{2}+e^{-2 s}\right), \quad i=2,3,
$$

for all $z \in \mathbb{R}$ and all $s \leq R$.

\section{Proof}

We may assume that $i=2$ since $f_{3}(s, z)=f_{2}(s,-z)$. Let $b \in \mathbb{R}$ be the solution to $b^{2}-b=e^{-4 R}$ that is larger than 1. We shall establish (6.10) for all $s \leq R$ with

$$
A=\frac{b-1}{b} \text {. }
$$

Inserting the definition of $f_{2}$ we see that, with this value of $A,(6.10)$ is equivalent to

$$
e^{2 s}(z+1)^{2}+e^{-2 s} \geq \frac{b-1}{b}\left(e^{2 s} z^{2}+e^{-2 s}\right)
$$

and hence also to

$$
e^{2 s}\left(z^{2}+2 b z+b\right)+e^{-2 s} \geq 0 .
$$

This last inequality is valid for all $z \in \mathbb{R}$ and $s \leq R$ since the minimum of $z^{2}+$ $2 b z+b$ as a function of $z$ is $-b^{2}+b=-e^{-4 R}$. 
Proof of Theorem 6.1

Let $\phi \in \mathcal{C}\left(X_{n}\right)$. Throughout the proof we will use the notation

$$
I_{\phi}(s):=\int_{U_{k, k}} \phi\left(a_{s} u\right) d u .
$$

The proof consists of three parts. In part (a) we will address the rapid decay of $e^{s} I_{\phi}(s)$ for $s \rightarrow-\infty$ for both $n$ odd and $n$ even. In part (b) we will address the similar decay for $s \rightarrow \infty$ when $n$ is even. Finally, in part (c) we will address the limit behavior for $s \rightarrow \infty$ when $n$ is odd.

Fix $N \in \mathbb{N}$. Then by (2.4), the function $\phi$ satisfies the estimate

$$
|\phi(x)| \leq \mu_{1, N}(\phi) f(x) \quad\left(x \in X_{n}\right)
$$

where $f: X_{n} \rightarrow \mathbb{R}_{>0}$ is given by

$$
f\left(k^{\prime} a_{t} \cdot H\right)=(2 \cosh (4 t))^{-\frac{n-1}{4}}(1+\log (2 \cosh (4 t)))^{-N} \quad\left(k^{\prime} \in K, t \in \mathbb{R}\right) .
$$

Let

$$
I_{f}(s):=\int_{U_{k, k}} f\left(a_{s} u \cdot H\right) d u .
$$

Then we have the estimate

$$
\left|I_{\phi}(s)\right| \leq \mu_{1, N}(\phi) I_{f}(s),
$$

so that for parts (a) and (b) it suffices to show that (6.1) and (6.2) are satisfied with $\phi$ replaced by $f$. Define $\Psi: \mathbb{R}_{\geq 1} \rightarrow \mathbb{R}$ by

$$
\Psi(r)=r^{-\frac{n-1}{4}}(1+\log (r))^{-N} \quad(r \geq 1, N \geq 0) .
$$

Then it follows that

$$
\Psi(2 \cosh (4 t))=f\left(k^{\prime} a_{t} \cdot H\right)
$$

for $k^{\prime} \in K$ and $t \in \mathbb{R}$. Moreover, using (6.4) we see that

$$
f\left(a_{s} u_{x, y, z}\right)=\Psi\left(f_{1}+f_{2}\|x\|^{2}+f_{3}\|y\|^{2}+\|x\|^{2}\|y\|^{2}\right) .
$$

Hence,

$$
I_{f}(s)=\int_{z \in \mathbb{R}} \int_{y \in \mathbb{R}^{k-2}} \int_{x \in \mathbb{R}^{n-k}} \Psi\left(f_{1}+f_{2}\|x\|^{2}+f_{3}\|y\|^{2}+\|x\|^{2}\|y\|^{2}\right) d x d y d z .
$$

Part (a). Performing the following substitutions on the inner integrals,

$$
x=\left(f_{1} / f_{2}\right)^{1 / 2} \xi, \quad y=\left(f_{1} / f_{3}\right)^{1 / 2} \eta,
$$

we obtain

$$
I_{f}(s)=\int_{z \in \mathbb{R}} \int_{\eta \in \mathbb{R}^{k-2}} \int_{\xi \in \mathbb{R}^{n-k}} \Psi\left(F(\xi, \eta) f_{1}\right)\left(\frac{f_{1}}{f_{2}}\right)^{\frac{n-k}{2}}\left(\frac{f_{1}}{f_{3}}\right)^{\frac{k-2}{2}} d \xi d \eta d z
$$

where

$$
F(\xi, \eta):=1+\|\xi\|^{2}+\|\eta\|^{2}+\frac{f_{1}}{f_{2} f_{3}}\|\xi\|^{2}\|\eta\|^{2}
$$


In the following we assume that $N \geq 6$. Using (6.13) we now see that the integrand in (6.15) is absolutely bounded by

$$
F(\xi, \eta)^{-\frac{n-1}{4}} f_{1}^{\frac{n-3}{4}} f_{2}^{-\frac{n-k}{2}} f_{3}^{-\frac{k-2}{2}}\left(1+\log F(\xi, \eta)+\log \left(f_{1}\right)\right)^{-N} .
$$

Let

$$
\epsilon=\frac{n+3}{2}-k
$$

Then $0 \leq \epsilon \leq 1$ by our assumption on $k$. Moreover,

$$
n-k=\frac{n-3}{2}+\epsilon, \quad k-2=\frac{n-3}{2}+1-\epsilon .
$$

Since $f_{1} \leq f_{2} f_{3}$, it follows that the expression (6.16) is bounded from above by

$$
F(\xi, \eta)^{-\frac{n-1}{4}} f_{2}^{-\frac{\epsilon}{2}} f_{3}^{-\frac{1-\epsilon}{2}}\left(1+\log F(\xi, \eta)+\log \left(f_{1}\right)\right)^{-N}
$$

for all $s$.

From (6.8) we infer that

$$
F(\xi, \eta) \geq\left(1+\frac{1}{2}\|\xi\|^{2}\right)\left(1+\frac{1}{2}\|\eta\|^{2}\right) .
$$

It thus finally follows that

$$
I_{f}(s) \leq I_{1} I_{2} I_{3}(s)
$$

where

$$
I_{1}=\int_{\mathbb{R}^{n-k}} g_{1}(\xi) d \xi, \quad I_{2}=\int_{\mathbb{R}^{k-2}} g_{2}(\eta) d \xi, \quad I_{3}(s)=\int_{\mathbb{R}} g_{3}(s, z) d z
$$

with

$$
\begin{gathered}
g_{1}(\xi)=\left(1+\frac{1}{2}\|\xi\|^{2}\right)^{-\frac{n-1}{4}}\left(1+\log \left(1+\frac{1}{2}\|\xi\|^{2}\right)\right)^{-2} \\
g_{2}(\eta)=\left(1+\frac{1}{2}\|\eta\|^{2}\right)^{-\frac{n-1}{4}}\left(1+\log \left(1+\frac{1}{2}\|\eta\|^{2}\right)\right)^{-2}, \\
g_{3}(s, z)=f_{2}(s, z)^{-\frac{\epsilon}{2}} f_{3}(s, z)^{-\frac{1-\epsilon}{2}}\left(1+\log \left(f_{1}(s, z)\right)\right)^{-(N-4)} .
\end{gathered}
$$

It follows from (6.17) that the dimensions $n-k$ and $k-2$ are at most $\frac{n-1}{2}$ so that $I_{1}$ and $I_{2}$ are finite, thanks to the logarithmic terms (which in fact are needed in at most one of the integrals). Thus, it only remains to estimate the third integral in $(6.19)$.

We first assume that $s \leq R$ for some given $R \in \mathbb{R}$. Using (6.10) for $f_{2}$ and $f_{3}$, and estimating two of the logarithmic factors in $I_{3}(s)$ by $(6.9)$ and the remaining ones by $f_{1} \geq \max \left\{2, e^{-4 s}\right\}$, we find

$I_{3}(s) \leq C \int_{\mathbb{R}}\left(e^{2 s} z^{2}+e^{-2 s}\right)^{-1 / 2}\left(1+\log \left(1+z^{2}\right)\right)^{-2} d z(1+\max \{\log 2,-4 s\})^{-(N-6)}$

for all $s \leq R$, with $C>0$ a constant depending on $N$. Substituting $z=e^{-2 s} \zeta$ and using that $s \leq R$, we find

$$
I_{3}(s) \leq C e^{-s} \int_{\mathbb{R}}\left(\zeta^{2}+1\right)^{-1 / 2}\left(1+\log \left(1+e^{-4 R} \zeta^{2}\right)\right)^{-2} d \zeta(1+|s|)^{-(N-6)},
$$


with a new constant $C>0$. The integral converges, and since $N$ was arbitrary we conclude from (6.18) that (6.2) holds, regardless of the parity of $n$. This completes part (a) of the proof.

Part (b). We assume that $s \geq 0$ and that $n$ is even. Then $k=\frac{n+2}{2}$ and $\epsilon=\frac{1}{2}$; hence,

$$
I_{3}(s)=\int_{\mathbb{R}} f_{2}(s, z)^{-\frac{1}{4}} f_{3}(s, z)^{-\frac{1}{4}}\left(1+\log \left(f_{1}(s, z)\right)\right)^{-(N-4)} d z .
$$

The integral over $\mathbb{R}$ can be replaced by an integral over $\mathbb{R}_{>0}$, because $f_{2}(s,-z)=$ $f_{3}(s, z)$ and $f_{1}(s,-z)=f_{1}(s, z)$. We split the integration into two parts and integrate separately over the interval $[1-\delta, 1+\delta]$ and its complement in $\mathbb{R}_{>0}$, with $\delta \in(0,1)$ to be fixed later. (It will depend on $s$.) Let us write $J_{\delta}(s)$ for the integral over $[1-\delta, 1+\delta]$ and ${ }^{c} J_{\delta}(s)$ for the integral over the complement of this set in $\mathbb{R}_{>0}$.

For $J_{\delta}(s)$ we use the estimates

$$
f_{1} \geq 2, \quad f_{2}(s, z) \geq e^{2 s}, \quad f_{3}(s, z) \geq e^{2 s}(z-1)^{2},
$$

for $z \geq 0$, and we obtain

$$
J_{\delta}(s) \leq e^{-s} \int_{1-\delta}^{1+\delta}|z-1|^{-1 / 2} d z=4 e^{-s} \delta^{1 / 2} .
$$

For ${ }^{c} J_{\delta}(s)$ we estimate $f_{1}$ by (6.9) in two of the logarithmic factors and by $f_{1} \geq e^{4 s} \delta^{2}$ in the remaining factors. Furthermore, we estimate

$$
f_{2}(s, z) \geq e^{2 s}(1+z)^{2}, \quad f_{3}(s, z) \geq e^{2 s}(z-1)^{2}
$$

and obtain

$$
\begin{aligned}
{ }^{c} J_{\delta}(s) \leq & C e^{-s} \int_{0}^{\infty}(1+z)^{-1 / 2}|z-1|^{-1 / 2} \\
& \times\left(1+\log \left(1+z^{2}\right)\right)^{-2} d z\left(1+\log \left(e^{4 s} \delta^{2}\right)\right)^{-(N-6)},
\end{aligned}
$$

with a constant $C>0$ depending on $N$ but independent of $s$ and $\delta$. The integral over $z$ converges, and we obtain (with a new constant $C>0$ of the same (in)dependency),

$$
{ }^{c} J_{\delta}(s) \leq C e^{-s}\left(1+\log \left(e^{4 s} \delta^{2}\right)\right)^{-(N-6)} .
$$

By adding (6.21) and (6.22), we see that by choosing $\delta=e^{-\frac{3}{2} s}$ we can ensure that

$$
I_{3}(s) \leq C e^{-s}(1+s)^{-(N-6)},
$$

with yet another constant $C>0$. This implies (6.1) for the remaining case $s \geq 0$.

Part (c). We now turn to the statements about the limit in Theorem 6.1. Assume that $n$ is odd. We shall first deal with the case $k=l=\frac{n+1}{2}$ and consider the integral 


$$
\begin{aligned}
I_{\phi}(s) & =\int_{U_{\frac{n+1}{2}, \frac{n+1}{2}}} \phi\left(a_{s} u \cdot H\right) d u \\
& =\int_{z \in \mathbb{R}} \int_{y \in \mathbb{R}^{\frac{n-3}{2}} \times\{0\}^{\frac{n-1}{2}}} \int_{x \in\{0\}^{\frac{n-3}{2}} \times \mathbb{R}^{\frac{n-1}{2}}} \phi\left(a_{s} u_{x, y, z}\right) d x d y d z .
\end{aligned}
$$

This time, we perform the substitution of variables

$$
x=e^{s} \xi, \quad y=e^{-s} \eta, \quad \text { and } \quad z=e^{-2 s} \omega-1
$$

and obtain from (6.23) that

$$
e^{s} I_{\phi}(s)=\int_{\omega \in \mathbb{R}} \int_{\eta \in \mathbb{R}^{\frac{n-3}{2}} \times\{0\}^{\frac{n-1}{2}}} \int_{\xi \in\{0\}^{\frac{n-3}{2}} \times \mathbb{R}^{\frac{n-1}{2}}} \Phi_{s}(\xi, \eta, \omega) d \xi d \eta d \omega,
$$

where

$$
\Phi_{s}(\xi, \eta, \omega)=\phi\left(a_{s} u_{e^{s} \xi, e^{-s} \eta, e^{-2 s} \omega-1}\right) .
$$

Recall the definition of $\kappa$ from (2.1). From Lemma 6.5 below we see that

$$
\lim _{s \rightarrow \infty} \Phi_{s}(\xi, \eta, \omega)=\phi\left(\kappa^{-1} v_{(\omega, \eta), \frac{2}{3} \xi}\right),
$$

for all $(\omega, \eta, \xi) \in \mathbb{R}^{n-1}$. Assuming that we may interchange the limit for $s \rightarrow \infty$ with the integral on the right-hand side of (6.25) we obtain

$$
\begin{aligned}
\lim _{s \rightarrow \infty} e^{s} I_{\phi}(s) & =\int_{\mathbb{R}} \int_{\mathbb{R}^{\frac{n-3}{2}}} \int_{\mathbb{R}^{\frac{n-1}{2}}} \phi\left(\kappa^{-1} v_{(\omega, \eta), \frac{2}{3} \xi}\right) d \xi d \eta d \omega \\
& =\int_{V_{\frac{n+1}{2}}} \phi\left(\kappa^{-1} v\right) d v,
\end{aligned}
$$

for the choice of Lebesgue measure $d v$ corresponding to $(2 / 3)^{\frac{n-1}{2}} d \xi d \eta d \omega$.

Thus, for the proof of (6.3) it remains to be shown that we may interchange the limit and integral in (6.25). To prove this, we adopt the following strategy.

For $0<\delta<1$ and $s>0$ we define the set

$$
A_{\delta, s}:=\mathbb{R}^{\frac{n-1}{2}} \times \mathbb{R}^{\frac{n-3}{2}} \times\left[(2-\delta) e^{2 s},(2+\delta) e^{2 s}\right]
$$

and denote by $B_{\delta, s}$ its complement in $\mathbb{R}^{n-1} \simeq \mathbb{R}^{\frac{n-1}{2}} \times \mathbb{R}^{\frac{n-3}{2}} \times \mathbb{R}$. We observe that for every $v \in \mathbb{R}^{n-1}$ there exists $s_{0} \in \mathbb{R}$ such that $v \in B_{\delta, s}$ for all $0<\delta<1$ and $s \geq s_{0}$. Accordingly, the characteristic function $1_{B_{\delta, s}}$ converges to the constant function 1 , pointwise on $\mathbb{R}^{n-1}$, for $s \rightarrow \infty$.

In the text below, we will show that

$$
\int_{A_{\delta, s}}\left|\Phi_{s}(\xi, \eta, \omega)\right| d \xi d \eta d \omega \leq C \delta
$$

for a suitable constant $C>0$, independent of $s$ and $\delta$. On the other hand, we will show that for each $0<\delta<1$ there exists an integrable function $F_{\delta}: \mathbb{R}^{n-1} \rightarrow \mathbb{R}_{\geq 0}$ such that

$$
1_{B(\delta, s)}\left|\Phi_{s}\right| \leq F_{\delta} \quad \text { on } \mathbb{R}^{n-1}
$$


for all $s>0$. By an application of Lebesgue's convergence theorem it then follows that

$$
\lim _{s \rightarrow \infty} \int_{B_{\delta, s}} \Phi_{s} d \xi d \eta d \omega=\int_{\mathbb{R}^{n}} \lim _{s \rightarrow \infty} \Phi_{s} d \xi d \eta d \omega
$$

Combining this with (6.26) we readily see that the interchange of the limit and integral is allowed.

To achieve the goals mentioned above, we recall the definitions of $f_{1}, f_{2}$, and $f_{3}$ from $(6.5)-(6.7)$, but now considered as functions of $(s, \omega)$. Then by virtue of the substitution (6.24), it follows from (6.6) that

$$
f_{2}=e^{-2 s}\left(\omega^{2}+1\right) \text {. }
$$

Furthermore, we define $f$ and $\Psi$ as in (6.12) and (6.13). Then from (6.11) and (6.14) we infer that

$$
\left|\Phi_{s}(\xi, \eta, \omega)\right| \leq \mu_{1, N}(\phi) \Psi\left(f_{1}+e^{2 s} f_{2}\|\xi\|^{2}+e^{-2 s} f_{3}\|\eta\|^{2}+\|\xi\|^{2}\|\eta\|^{2}\right) .
$$

Since $\Psi$ is decreasing on $\mathbb{R}_{\geq 1}$, whereas $f_{3} \geq f_{1} / f_{2}$ by (6.8), it follows that

$$
\left|\Phi_{s}(\xi, \eta, \omega)\right| \leq \mu_{1, N}(\phi) \Psi_{s}(\xi, \eta, \omega)
$$

where

$$
\Psi_{s}(\xi, \eta, \omega):=\Psi\left(f_{1}+e^{2 s} f_{2}\|\xi\|^{2}+\frac{f_{1}}{e^{2 s} f_{2}}\|\eta\|^{2}+\|\xi\|^{2}\|\eta\|^{2}\right) .
$$

This estimate, combined with (6.28), motivates the use of a final substitution

$$
\xi=\left(1+\omega^{2}\right)^{-\frac{1}{2}} \chi, \quad \eta=\left(1+\omega^{2}\right)^{\frac{1}{2}} \psi .
$$

Note that this substitution does not affect the subsets $A(\delta, s)$ and $B(\delta, s)$ of $\mathbb{R}^{n-1}$, defined above. For any measurable subset $S \subseteq \mathbb{R}$, the function $\Psi_{s}$ is integrable over $\mathbb{R}^{n-2} \times S$ if and only if the function

$$
\widetilde{\Psi}_{s}(\chi, \psi, \omega):=\left(1+\omega^{2}\right)^{-\frac{1}{2}} \Psi\left(f_{1}+\|\chi\|^{2}+f_{1}\|\psi\|^{2}+\|\chi\|^{2}\|\psi\|^{2}\right)
$$

is integrable over this set, and accordingly,

$$
\int_{\mathbb{R}^{n-2} \times S} \Psi_{s}(\xi, \eta, \omega) d \xi d \eta d \omega=\int_{\mathbb{R}^{n-2} \times S} \widetilde{\Psi}_{s}(\chi, \psi, \omega) d \chi d \psi d \omega .
$$

We observe that

$$
1+\|\chi\|^{2}+\|\psi\|^{2}+\|\chi\|\|\psi\| \geq\left(1+\frac{1}{2}\|\chi\|\right)\left(1+\frac{1}{2}\|\psi\|\right) .
$$

Since $\Psi: r \mapsto r^{-\frac{n-1}{4}}(1+\log r)^{-N}$ is decreasing and $f_{1} \geq 2$, we now obtain the estimate

$$
\begin{aligned}
\widetilde{\Psi}(\chi, \psi, \omega) & \leq\left(1+\omega^{2}\right)^{-1 / 2} \Psi\left(f_{1}+\|\chi\|^{2}+\|\psi\|^{2}+\|\chi\|^{2}\|\psi\|^{2}\right) \\
& \leq g_{1}(\chi) g_{2}(\psi) h_{s}(\omega),
\end{aligned}
$$

where $g_{1}, g_{2}$ are defined as in (6.20) and where

$$
h_{s}(\omega):=(1+\omega)^{-\frac{1}{2}}\left(1+\log f_{1}\right)^{-(N-4)} .
$$


We observed already that the functions $g_{1}$ and $g_{2}$ are integrable over $\mathbb{R}^{\frac{n-1}{2}}$ and $\mathbb{R}^{\frac{n-3}{2}}$, with integrals $I_{1}$ and $I_{2}$, respectively. Let us therefore focus on the function $h_{s}$. On the interval $\left[(2-\delta) e^{2 s},(2+\delta) e^{2 s}\right]$ we have the estimates $1+\omega^{2} \geq e^{4 s}$ and $f_{1} \geq 1$. Hence,

$$
\int_{I_{\delta, s}} h_{s}(\omega) d \omega \leq \int_{(2-\delta) e^{2 s}}^{(2+\delta) e^{2 s}} e^{-2 s} d \omega=2 \delta
$$

It follows that

$$
\int_{A_{\delta, s}} \Psi_{s}(\xi, \eta, \omega) d \xi d \eta d \omega \leq 2 I_{1} I_{2} \delta,
$$

and by applying (6.29) we obtain the estimate (6.26).

It remains to prove the claimed majorization of $\Phi_{s}$ on $B_{\delta, s}$. Here we shall use the following lower bound on $f_{1}$ :

$$
f_{1} \geq 1+\frac{1}{2} \delta^{2} \omega^{2} \quad\left(\omega \notin\left[(2-\delta) e^{2 s},(2+\delta) e^{2 s}\right]\right) .
$$

To see this, note that the condition on $\omega$ is equivalent to $\left|2-e^{-2 s} \omega\right| \geq \delta$, and hence (6.30) follows immediately from the estimates $f_{1} \geq \frac{1}{2} f_{2} f_{3}$ and $f_{1} \geq 2$ (see $(6.8))$.

From (6.30) we obtain the estimate

$$
h_{s}(\omega) \leq\left(1+\omega^{2}\right)^{-\frac{1}{2}}\left(1+\frac{1}{2} \delta^{2} \omega^{2}\right)^{-(N-4)} \quad\left(\omega \in \mathbb{R} \backslash\left[(2-\delta) e^{2 s},(2+\delta) e^{2 s}\right]\right)
$$

for every $s>0$. We now make the additional assumption that $N \geq 5$ to ensure that the function on the right-hand side is integrable over $\mathbb{R}$.

Define the function $\widetilde{G}_{\delta}: \mathbb{R}^{n-1} \rightarrow \mathbb{R}_{\geq 0}$ by

$$
\widetilde{G}_{\delta}(\chi, \psi, \omega)=g_{1}(\chi) g_{2}(\psi)\left(\omega^{2}+1\right)^{-\frac{1}{2}}\left(1+\log \left(1+\frac{1}{2} \delta^{2} \omega^{2}\right)\right)^{-(N-4)} .
$$

Then $\widetilde{G}_{\delta}$ is integrable on $\mathbb{R}^{n-1}$, and for every $s>0$ we have the estimate

$$
\widetilde{\Psi}_{s} \leq \widetilde{G}_{\delta} \quad \text { on } B_{\delta, s} .
$$

Define $G_{\delta}: \mathbb{R}^{n-1} \rightarrow \mathbb{R}_{\geq 0}$ by

$$
G_{\delta}(\xi, \eta, \omega)=\left(1+\omega^{2}\right)^{\frac{1}{2}} \widetilde{G}_{\delta}\left(\left(1+\omega^{2}\right)^{\frac{1}{2}} \xi,\left(1+\omega^{2}\right)^{-\frac{1}{2}} \eta, \omega\right) .
$$

Then $G_{\delta}$ is integrable on $\mathbb{R}^{n-1}$, and it follows that

$$
\Psi_{s} \leq G_{\delta} \quad \text { on } B_{\delta, s}
$$

for every $s>0$. In view of (6.29) this proves (6.27) with $F_{\delta}:=\mu_{1, N}(\phi) G_{\delta}$. We have thus established the limit formula (6.3) for the case $k=l=\frac{n+1}{2}$.

We will complete the proof of Theorem 6.1 by proving (6.3) for the remaining case $k=l=\frac{n+3}{2}$. Let $w$ be the longest Weyl group element for the root system $\Sigma_{\mathfrak{h}}=\Sigma\left(Z_{\mathfrak{h}}\left(\mathfrak{a}_{\mathfrak{q}}\right) ; \mathfrak{a}_{\mathfrak{h}}\right)$, relative to the positive system (4.1), and let $w_{0} \in N_{K \cap H}(\mathfrak{a}) \cap$ $Z_{K \cap H}\left(\mathfrak{a}_{\mathfrak{q}}\right)$ be a representative for $w$. By Lemma 4.7 , 


$$
\begin{aligned}
\lim _{s \rightarrow \infty} e^{s} \int_{U_{\frac{n+3}{2}, \frac{n+3}{2}}} \phi\left(a_{s} u\right) d u & =\lim _{s \rightarrow \infty} e^{s} \int_{U_{\frac{n+1}{2}, \frac{n+1}{2}}} \phi\left(w_{0} \sigma \theta\left(a_{s} u\right)\right) d u \\
& =\int_{V_{\frac{n+1}{2}}} \phi\left(w_{0} \sigma \theta\left(\kappa^{-1} v\right) \cdot H\right) d v \\
& =\int_{\theta V_{\frac{n+1}{2}}} \phi\left(w_{0} \kappa \bar{v} \cdot H\right) d \bar{v} .
\end{aligned}
$$

Let $w_{1}$ be a representative in $N_{K \cap H}(\mathfrak{b})$ for the longest Weyl group element of $\Sigma_{\mathfrak{h}}(\mathfrak{b})$, relative to the positive system (5.1). Then, in view of Proposition 5.3,

$$
w_{1} \theta V_{\frac{n+1}{2}} w_{1}^{-1}=V_{\frac{n+1}{2}} .
$$

Hence, the integral in (6.31) is equal to

$$
\int_{V_{\frac{n+1}{2}}} \phi\left(w_{0} \kappa w_{1}^{-1} v \cdot H\right) d v .
$$

This completes the proof of Theorem 6.1.

\section{LEMMA 6.5}

Let $k=l=\frac{n+1}{2}$. Then with notation as in (4.9) and (5.5) and writing $u(x, y, z)=$ $u_{x, y, z}$, we have the following limit in $X_{n}$ :

$$
\lim _{s \rightarrow \infty} a_{s} u\left(e^{s} \xi, e^{-s} \eta, e^{-2 s} \omega-1\right) \cdot H=\kappa^{-1} v_{(\omega, \eta), \frac{2}{3} \xi} \cdot H .
$$

Proof

We start with a computation in $\operatorname{SL}(2, \mathbb{R})$, whose Lie algebra has the standard Iwasawa decomposition $\mathfrak{s l}(2, \mathbb{R})=\mathbb{R} U \oplus \mathbb{R} Y \oplus \mathbb{R} V$, with

$$
U:=\left(\begin{array}{cc}
0 & -1 \\
1 & 0
\end{array}\right), \quad Y:=\left(\begin{array}{cc}
1 & 0 \\
0 & -1
\end{array}\right), \quad V:=\left(\begin{array}{cc}
0 & 1 \\
0 & 0
\end{array}\right) .
$$

We agree to write $k_{\varphi}:=\exp \varphi U, a_{s}:=\exp s Y$, and $n_{z}:=\exp z V$. In particular,

$$
\kappa:=k_{\frac{\pi}{4}}=\frac{1}{\sqrt{2}}\left(\begin{array}{cc}
1 & -1 \\
1 & 1
\end{array}\right) \quad \text { and } \quad n_{-1}=\left(\begin{array}{cc}
1 & -1 \\
0 & 1
\end{array}\right) .
$$

Let $\sigma$ be the involution of $\operatorname{SL}(2, \mathbb{R})$ given by switching the diagonal entries as well as the off-diagonal entries, and let $H_{0}$ denote the associated group of fixed points.

We will first show that in the quotient $\operatorname{SL}(2, \mathbb{R}) / H_{0}$ we have

$$
\lim _{s \rightarrow \infty} a_{s} n_{-1} \cdot H_{0}=\kappa^{-1} \cdot H_{0} .
$$

According to [13, Theorem 1.3] the map $G \rightarrow G, g \mapsto g \sigma(g)^{-1}$ induces a diffeomorphism from $G / G^{\sigma}$ onto a submanifold of $G$. Applying this general fact to the situation at hand, we see that, for (6.32) to be valid, it suffices to show that

$$
a_{s} n_{-1} \sigma\left(a_{s} n_{-1}\right)^{-1} \rightarrow \kappa \sigma(\kappa)^{-1}=k_{\frac{\pi}{2}} \quad(s \rightarrow \infty) .
$$

Now this follows by a straightforward calculation. 
In turn, it follows from (6.32) that there exists a function $q: \mathbb{R} \rightarrow \mathrm{SL}(2, \mathbb{R})$ with $\lim _{s \rightarrow \infty} q(s)=e$ and

$$
n_{1} a_{-s} \kappa^{-1} q(s) \in H_{0} \quad(s \in \mathbb{R}) .
$$

We agree to identify $\operatorname{SL}(2, \mathbb{R})$ with a closed subgroup of $\operatorname{SL}(n, \mathbb{R})$ via the embedding

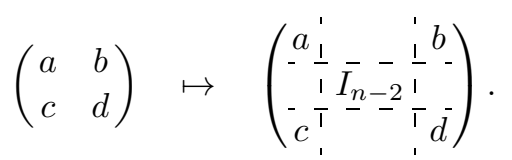

Then $\kappa$ and $a_{s}$ in $\operatorname{SL}(2, \mathbb{R})$ correspond with the similarly denoted elements in $\mathrm{SL}(n, \mathbb{R})$. Furthermore, $H_{0}$ equals the intersection of $\mathrm{SL}(2, \mathbb{R})$ with the subgroup $H$ of $\operatorname{SL}(n, \mathbb{R})$.

For $x \in\{0\}^{\frac{n-3}{2}} \times \mathbb{R}^{\frac{n-1}{2}}, y \in \mathbb{R}^{\frac{n-3}{2}} \times\{0\}^{\frac{n-1}{2}}$, and $z \in \mathbb{R}$ we define

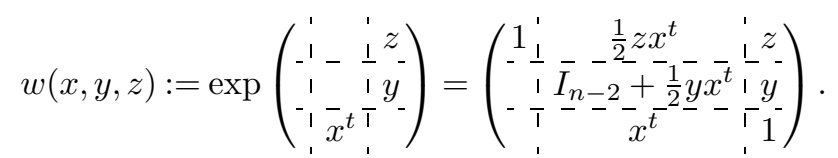

Then by a straightforward calculation, one checks that the matrix

$$
A=w(x, y, z)^{-1} u\left(\left(1-\frac{1}{2} z\right) x, y, z\right)
$$

satisfies $S A S^{-1}=A$ and, hence, belongs to $H$. Thus, if $z \neq 2$, then

$$
\begin{aligned}
a_{s} u(x, y, z) \cdot H & =a^{s} w\left(\left(1-\frac{1}{2} z\right)^{-1} x, y, z\right) \cdot H \\
& =a^{s} w\left(\left(1-\frac{1}{2} z\right)^{-1} x, y, z\right) n_{1} a_{-s} \kappa^{-1} q(s) \cdot H \\
& =a^{s} w\left(\left(1-\frac{1}{2} z\right)^{-1} x, y, z+1\right) a_{s}^{-1} \kappa^{-1} q(s) \cdot H \\
& =w\left(\left(1-\frac{1}{2} z\right)^{-1} e^{-s} x, e^{s} y, e^{2 s}(z+1)\right) \kappa^{-1} q(s) \cdot H .
\end{aligned}
$$

Through the substitution (6.24) the last expression becomes

$$
w\left(2\left(3-e^{-2 s} \omega\right)^{-1} \xi, \eta, \omega\right) \kappa^{-1} q(s) \cdot H .
$$

For $s \rightarrow \infty$ this expression tends to (see (6.33) and (5.5))

$$
w\left(\frac{2}{3} \xi, \eta, \omega\right) \kappa^{-1} \cdot H=\kappa^{-1} v_{(\omega, \eta), \frac{2}{3} \xi} \cdot H
$$

The result follows.

Acknowledgments. We would like to thank Mogens Flensted-Jensen for many fruitful discussions related to the present work. Kuit's work is supported by the Danish National Research Foundation through the Centre for Symmetry and Deformation (DNRF92). 


\section{References}

[1] N. B. Andersen, M. Flensted-Jensen, and H. Schlichtkrull, Cuspidal discrete series for semisimple symmetric spaces, J. Funct. Anal. 263 (2012), no. 8, 2384-2408. MR 2964687. DOI 10.1016/j.jfa.2012.07.009.

[2] D. Bălibanu and E. P. van den Ban, Convexity theorems for semisimple symmetric spaces, Forum Math. 28 (2016), no. 6, 1167-1204. MR 3567864. DOI 10.1515/forum-2015-0079.

[3] E. P. van den Ban, The principal series for a reductive symmetric space, II: Eisenstein integrals, J. Funct. Anal. 109 (1992), no. 2, 331-441. MR 1186325. DOI 10.1016/0022-1236(92)90021-A.

[4] E. P. van den Ban and J. J. Kuit, Cusp forms for reductive symmetric spaces of split rank one, Represent. Theory 21 (2017), 467-533. MR 3723155. DOI $10.1090 /$ ert/507.

[5] Y. Benoist, Analyse harmonique sur les espaces symétriques nilpotents, J. Funct. Anal. 59 (1984), no. 2, 211-253. MR 0766490. DOI 10.1016/0022-1236(84)90073-9.

[6] G. van Dijk and M. Poel, The Plancherel formula for the pseudo-Riemannian space $\mathrm{SL}(n, \mathbf{R}) / \mathrm{GL}(n-1, \mathbf{R})$, Compos. Math. 58 (1986), no. 3, 371-397. MR 0846911.

[7] $\quad$ The irreducible unitary $\mathrm{GL}(n-1, \mathbf{R})$-spherical representations of SL $(n, \mathbf{R})$, Compos. Math. 73 (1990), no. 1, 1-30. MR 1042452.

[8] S. Helgason, Differential Geometry and Symmetric Spaces, Pure Appl. Math. 12, Academic Press, New York, 1962. MR 0145455.

[9] A. G. Helminck and S. P. Wang, On rationality properties of involutions of reductive groups, Adv. Math. 99 (1993), no. 1, 26-96. MR 1215304. DOI 10.1006/aima.1993.1019.

[10] A. W. Knapp, Lie Groups Beyond an Introduction, 2nd ed., Progr. Math. 140, Birkhäuser Boston, Boston, 2002. MR 1920389.

[11] M. T. Kosters and G. van Dijk, Spherical distributions on the pseudo-Riemannian space $\operatorname{SL}(n, \mathbf{R}) / \mathrm{GL}(n-1, \mathbf{R})$, J. Funct. Anal. 68 (1986), no. 2, 168-213. MR 0852659. DOI 10.1016/0022-1236(86)90004-2.

[12] W. A. Kosters, Eigenspaces of the Laplace-Beltrami-operator on $\mathrm{SL}(n, \mathbf{R}) / \mathrm{S}(\mathrm{GL}(1) \times \mathrm{GL}(n-1)), I$, Nederl. Akad. Wetensch. Indag. Math. 47 (1985), no. 1, 99-123. MR 0783010. II, no. 2, 125-145. MR 0799076.

[13] O. Loos, Symmetric Spaces, I: General Theory, W. A. Benjamin, New York, 1969. MR 0239005.

[14] T. Matsuki, The orbits of affine symmetric spaces under the action of minimal parabolic subgroups, J. Math. Soc. Japan 31 (1979), no. 2, 331-357. MR 0527548. DOI 10.2969/jmsj/03120331.

[15] G. D. Mostow, Fully reducible subgroups of algebraic groups, Amer. J. Math. 78 (1956), 200-221. MR 0092928. DOI 10.2307/2372490. 
[16] H. Ochiai, Invariant distributions on a non-isotropic pseudo-Riemannian symmetric space of rank one, Indag. Math. (N.S.) 16 (2005), nos. 3-4, 631-638. MR 2313641. DOI 10.1016/S0019-3577(05)80043-6.

[17] W. Rossmann, The structure of semisimple symmetric spaces, Canad. J. Math. 31 (1979), no. 1, 157-180. MR 0518716. DOI 10.4153/CJM-1979-017-6.

van den Ban: Mathematical Institute, Utrecht University, Utrecht, The Netherlands; E.P.vandenBan@uu.nl

Kuit: Institut für Mathematik, Universität Paderborn, Paderborn, Germany; j.j.kuit@gmail.com

Schlichtkrull: Department of Mathematical Sciences, University of Copenhagen, Copenhagen, Denmark; schlicht@math.ku.dk 